\title{
Brane current algebras and generalised geometry from QP manifolds. Or, "when they go high, we go low"
}

\section{Alex S. Arvanitakis}

Theoretische Natuurkunde, Vrije Universiteit Brussel, and the International Solvay Institutes, Pleinlaan 2, B-1050 Brussels, Belgium

E-mail: alex.s.arvanitakis@vub.be

ABstract: We construct a Poisson algebra of brane currents from a QP-manifold, and show their Poisson brackets take a universal geometric form. This generalises a result of Alekseev and Strobl on string currents and generalised geometry to include branes with worldvolume gauge fields, such as the D3 and M5. Our result yields a universal expression for the 't Hooft anomaly that afflicts isometries in the presence of fluxes. We determine the current algebra in terms of (exceptional) generalised geometry, and show that the tensor hierarchy gives rise to a brane current hierarchy. Exceptional complex structures produce pairs of anomaly-free current subalgebras on the M5-brane worldvolume.

KEYwords: Bosonic Strings, BRST Quantization, Differential and Algebraic Geometry, Superspaces

ArXiv ePrint: 2103.08608 


\section{Contents}

1 Introduction and discussion $\quad 1$

1.1 Main result 3

1.2 Examples 5

2 The universal bracket formula and the brane phase space construction 7

2.1 On perversion

3 Examples of the brane phase space construction

$\begin{array}{ll}3.1 & \text { Degree } P=1 \text { QP manifolds and the Schouten bracket }\end{array}$

3.2 Generic $p$-brane without worldvolume gauge fields 11

3.2.1 F1 brane, or the Alekseev-Strobl current algebra $(p=1) \quad 16$

$\begin{array}{lll}3.2 .2 & \text { M2 brane } & 16\end{array}$

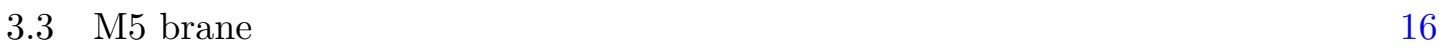

$\begin{array}{lll}3.4 & \text { D3 brane } & 21\end{array}$

4 Applications $\quad 24$

4.1 Brane dynamics 24

4.1.1 The spatial diffeomorphism constraints 24

4.1.2 The hamiltonian constraint 26

$\begin{array}{lll}4.2 & \text { Dirac structures and the 't Hooft anomaly } & 27\end{array}$

4.2.1 SUSY backgrounds and exceptional (generalised) complex structures 30

$4.3 \quad L_{\infty}$-algebras, brane currents, and the tensor hierarchy 31

A Sign manifesto, conventions, the AKSZ construction, etc. 33

\section{Introduction and discussion}

Consider the phase space of a closed bosonic string. It is spanned by the position $X^{\mu}(t, \sigma)$ and momentum $P_{\mu}(t, \sigma)(\sigma \in[0,2 \pi], \mu=0,1,2, \ldots d-1)$, that satisfy canonical equal-time Poisson brackets

$$
\left\{X^{\mu}\left(\sigma_{1}\right), P_{\nu}\left(\sigma_{2}\right)\right\}=\delta_{\nu}^{\mu} \delta\left(\sigma_{1}-\sigma_{2}\right) .
$$

For the string-theory string propagating on a metric and $B$-field background, the Virasoro constraints are expressed through $O(d, d)$-covariant quantities: the generalised metric $\mathcal{M}_{M N}$ containing the metric and $B$-field, the $O(d, d)$ structure $\eta_{M N}$, and the expression

$$
Z_{M} \equiv\left(\begin{array}{c}
\partial_{\sigma} X^{\mu} \\
P_{\mu}
\end{array}\right)
$$


in terms of which the phase-space or hamiltonian form of the action is

$$
S[X, P ; e, u]=\int d t d \sigma \partial_{t} X^{\mu} P_{\mu}-e \mathcal{M}^{M N} Z_{M} Z_{N}-u \eta^{M N} Z_{M} Z_{N},
$$

where $u, e$ are lagrange multipliers that enforce the worldsheet spatial diffeomorphism $\left(\eta^{M N} Z_{M} Z_{N}=0\right)$ and hamiltonian $\left(\mathcal{M}^{M N} Z_{M} Z_{N}=0\right)$ constraints; the Virasoro constraints are their sums and differences. This appearance of $O(d, d)$ in the hamiltonian form of worldsheet dynamics is an old observation due to Siegel [1]. We emphasise here that the background is arbitrary; it is not necessarily a torus. (For tori, of course, the observation is older.)

Alekseev and Strobl observed much later [2] that there is a distinguished class of currents involving the same expressions:

$$
J(v, \alpha)(\sigma) \equiv v^{\mu}(X(\sigma)) P_{\mu}(\sigma)+\alpha_{\mu}(X(\sigma)) \partial_{\sigma} X(\sigma) .
$$

Here $v$ is a vector field and $\alpha$ is a 1 -form on the background geometry $M$. They are distinguished because their Poisson brackets resolve into geometric expressions (in the tensors $(v, u, \alpha, \beta)$ on $M)$ :

$$
\begin{aligned}
\left\{J(v, \alpha)\left(\sigma_{1}\right), J(u, \beta)\left(\sigma_{2}\right)\right\} & =-J(w, \gamma) \delta\left(\sigma_{1}-\sigma_{2}\right)+\left(\iota_{u} \alpha+\iota_{v} \beta\right)\left(X\left(\sigma_{2}\right)\right) \partial_{\sigma_{1}} \delta\left(\sigma_{1}-\sigma_{2}\right) \\
w & =\mathcal{L}_{v} u, \quad \gamma=\mathcal{L}_{v} \beta-\mathcal{L}_{u} \alpha+d\left(\iota_{u} \alpha\right) .
\end{aligned}
$$

In fact the vector $w$ and 1 -form $\gamma$ in the resulting current $J(w, \gamma)$ are given by the Dorfman bracket on the generalised tangent bundle $T M \oplus T^{\star} M$, while the anomalous or Schwinger term involves the natural $O(d, d)$ structure of $T M \oplus T^{\star} M$. Alongside the obvious projection $T M \oplus T^{\star} M \rightarrow T M$, these define the data of a Courant algebroid [3, 4]. By incorporating the effect of a closed NS 3-form $H$ in the Poisson brackets, Alekseev-Strobl also showed they can recover arbitrary exact Courant algebroids (classified by the class of $H$ in $H_{\text {de Rham }}^{3}(M)$ [5]) from the current algebra.

Here, we generalise the Alekseev-Strobl picture by reversing their implication: for every algebroid structure of a certain type on a manifold $M$ (roughly, a Leibniz algebroid with extra data), we assign a Poisson algebra of currents in a brane sigma model on $M$. Less roughly, the algebroids are those that have a QP-manifold formulation: a graded manifold $\mathcal{M}$ with base $M$, a vector field $Q$ with $Q^{2}=0$, and a graded symplectic form $\omega$. QP manifolds also appear in the AKSZ construction of topological field theories [6], which we will employ (or rather, a minor generalisation thereof). A "QP" approach to current algebras was also pursued by Ikeda and $\mathrm{Xu}$ [7], who constructed a current algebra in the special case where $\mathcal{M}$ is a shifted cotangent bundle of another graded manifold $\mathcal{M}^{\prime}$ :

$$
\mathcal{M}=T^{\star}[P] \mathcal{M}^{\prime}
$$

(We were also notified of reference [8] in the same context, again for the cotangent case $\mathcal{M}=T^{\star}[P] \mathcal{M}^{\prime}$.) In our construction, we lift this restriction on $\mathcal{M}$. This in particular allows us to talk about branes with self-dual worldvolume gauge fields, like the M5 brane. 
The new ingredient in our construction is the apparently obscure zero-locus reduction (ZL reduction) for QP manifolds, due to Grigoriev, Semikhatov, and Tipunin [9]. The ZL reduction produces the Poisson bracket from the AKSZ construction and also selects a distinguished class of currents, analogous to the Alekseev-Strobl string currents. These correspond to the same bundles that appear in the $L_{\infty}$-algebra associated to the QP manifold. For those QP manifolds that capture exceptional generalised geometry [10], the distinguished currents are therefore associated to the tensor hierarchy, and their Poisson brackets are governed by the "exceptional" analogs of Courant/Dorfman brackets.

The last fact has been empirically observed (through direct calculations of Poisson brackets) in a number of works that discuss brane current algebras in phase space, starting with a relatively early paper by Bonelli and Zabzine that relates a $p$-brane current algebra to an algebroid involving $T M \oplus \Lambda^{p} T^{\star} M$ [11], and more recently for M2, M5 and D-branes by Hatsuda and collaborators [12-14] and also by Sakatani and Uehara [15]. Our construction explains why $O(d, d)$ - and $E_{d(d)}$-covariant structures and (exceptional) Dorfman, Courant, Vinogradov ... brackets were encountered on the brane phase space, and in some cases generalises the bracket formulas to accommodate the effects of the Wess-Zumino coupling to background fluxes. (This includes the cases of the M5 and D3 branes, to our knowledge.)

We emphasise that in the QP constructions in this paper there appear no "extended coordinates" corresponding to brane winding modes, because there appears to be some tension between $Q^{2}=0$ and extended coordinates [16-18]. Works including extended coordinates in the hamiltonian formalism on the brane include Sakatani and Uehara [19, 20], Linch and Siegel [21, 22] and the very recent paper of Osten [23] that focusses on the realisation of exceptional generalised geometry on brane currents. Another perspective complementary to ours is by Strickland-Constable [24], where the brane equations of motion on phase space are interpreted as geodesic equations for (exceptional) generalised geometry (see also $[25,26]$ ).

\subsection{Main result}

To state the result we need a few ingredients, similar to those of the AKSZ construction. We need a Q-manifold $\mathcal{N}$ for the brane worldvolume, and the QP manifold $\mathcal{M}$ for the target space:

- $(\mathcal{M}, \omega, Q)$ is a QP manifold with Q-structure $Q$ and compatible symplectic form $\omega$ of integer degree $P>0$ (and thus also a Poisson bracket $(\bullet, \bullet)$ of degree $-P$ ),

- $(\mathcal{N}, D)$ is a Q-manifold of compact base $\Sigma$ and Q-structure $D . \mathcal{N}$ must also have a (non-degenerate) measure $\int_{\mathcal{N}}: C^{\infty}(\mathcal{N}) \rightarrow \mathbb{R}$ of degree $1-P$, that satisfies "integration by parts":

$$
\int_{\mathcal{N}} D \epsilon=0 \quad \forall \epsilon \in C^{\infty}(\mathcal{N})
$$

Both Q structures have degree 1, as usual. We also impose the technical condition that all coordinates of $\mathcal{M}$ and $\mathcal{N}$ have deg $\geq 0$. They must also have Grassmann parity deg mod 2, except in subsection 3.1. (With both conditions, they are N-manifolds in the sense of Ševera [27].) 
In the AKSZ construction the degree of $\int_{\mathcal{N}}$ would be $-P-1$. We thus need a minor generalisation. All of the usual facts are true, modulo sign differences. In particular, the $\mathrm{Q}$ structures $D$ and $Q$ both give rise to a pair of $\mathrm{Q}$ structures $\bar{D}$ and $\bar{Q}$ on the supermanifold of mappings

$$
\operatorname{Maps}(\mathcal{N} \rightarrow \mathcal{M})
$$

that anticommute:

$$
[\bar{D}, \bar{Q}] \equiv \bar{D} \bar{Q}+\bar{Q} \bar{D}=0 .
$$

We now come to the general definition of the currents. For every function $f \in C^{\infty}(\mathcal{M})$, we can write down a current $\langle f|$, that maps "test" functions $\epsilon \in C^{\infty}(\mathcal{N})$ to scalar functionals: if $\varphi \in \operatorname{Maps}(\mathcal{N} \rightarrow \mathcal{M})$, we define

$$
\langle f \mid \epsilon\rangle(\varphi) \equiv(-1)^{P} \int_{\mathcal{N}}\left(\varphi^{\star} f\right) \epsilon
$$

for the scalar functional $\langle f \mid \epsilon\rangle \in C^{\infty}(\operatorname{Maps}(\mathcal{N} \rightarrow \mathcal{M}))$. We will write the current as

$$
\langle f \mid \epsilon\rangle \equiv(-1)^{P} \int_{\mathcal{N}} \boldsymbol{f} \epsilon,
$$

where boldface is used for superfields (which are produced by the pullback $\varphi^{\star}$ ).

The main result is the degree 0 (i.e. super) Poisson bracket structure and the explicit formula for the Poisson brackets of currents (1.10):

Proposition 1. There is a degree 0 Poisson bracket $\{\bullet, \bullet\}$ on the "zero locus" $\mathcal{Z}_{\bar{D}-\bar{Q}}$ of $\bar{D}-\bar{Q}$, where $\mathcal{Z}_{\bar{D}-\bar{Q}}$ is defined by the following quotient modulo the ideal $\mathcal{I}_{\bar{D}-\bar{Q}}$ generated by $\bar{D}-\bar{Q}$ :

$$
\mathcal{Z}_{\bar{D}-\bar{Q}} \cong C^{\infty}(\operatorname{Maps}(\mathcal{N} \rightarrow \mathcal{M})) / \mathcal{I}_{\bar{D}-\bar{Q}} .
$$

The bracket of two currents $\langle f|,\langle g|$ of the form (1.10) associated to $f, g \in C^{\infty}(\mathcal{M})$ is

$$
\{\langle f \mid \epsilon\rangle,\langle g \mid \eta\rangle\}=(-1)^{\epsilon(g+P+1)}\langle(f, Q g) \mid \epsilon \eta\rangle+(-1)^{\epsilon(g+P)+g}\langle(f, g) \mid \epsilon D \eta\rangle,
$$

where the $Z L$ quotient is implied.

We will, somewhat abusively, call the zero locus $\mathcal{Z}_{\bar{D}-\bar{Q}}$ the brane phase space or just phase space in all cases. We will also call the last term in (1.12) the Schwinger term due to the $D \eta$; when the test forms $\epsilon, \eta$ are stripped, it gives rise to a derivative of the Dirac delta function.

\section{Remarks.}

1. The currents (1.10) (and their linear combinations) are closed under the Poisson bracket.

2. The bracket (1.12) can be seen as a BFV bracket that is compatible with the degree assignments (if we identify $\operatorname{deg}=\mathrm{gh}$ ). In particular it is antisymmetric for even 
arguments, contrary to appearances. This is guaranteed by the ZL reduction along with integration by parts (1.6).

3. Since the Poisson bracket is of zero degree/ghost number, the degree-zero subspace is distinguished by virtue of being closed under Poisson brackets. The only currents (1.10) of degree zero are the ones arising from functions $f \in C^{\infty}(\mathcal{M})$ of degree less than $P$ :

$$
\operatorname{deg}\langle f \mid \epsilon\rangle=f+\epsilon+1-P=0 \Longrightarrow \operatorname{deg} f \leq P-1, \operatorname{deg} \epsilon \leq P-1,
$$

since $\mathcal{M}$ and $\mathcal{N}$ were both assumed to be $\mathrm{N}$-manifolds (all degrees non-negative). This is precisely the vector space where the $L_{\infty}$-algebra canonically associated to the QP manifold $\mathcal{M}$ lives [28]. We elaborate on this in section 4.3.

4. We do not deal with the technical issues of infinite-dimensional graded or supermanifolds such as $\operatorname{Maps}(\mathcal{N} \rightarrow \mathcal{M})$. (These are in principle surmountable e.g. via the theory of Schwarz and Konechny [29]; also see [30] specifically for Maps). Therefore we prove the Proposition in local charts, which is anyway necessary to pin down signs and factors. The constructions in this paper can be used as local models which are to be patched together with transition functions: we can cover the underlying manifold $M$ with open sets $U_{\alpha}$ such that $(\mathcal{M}, Q, \omega)$ takes the specific form employed in this paper, where the transition functions are symplectomorphisms. At least for $p=1, \mathcal{N}=T[1] \Sigma=T[1] S^{1}$, we imagine proceeding as follows: the free loop space $\mathcal{L} M \equiv \operatorname{Maps}\left(S^{1} \rightarrow M\right)$ can be covered by sets $\mathcal{L} U_{\alpha},{ }^{1}$ and symplectomorphisms of $\mathcal{M}$ lift to ones of $\operatorname{Maps}(T[1] \Sigma \rightarrow \mathcal{M})$. This should produce a construction of $\operatorname{Maps}\left(T[1] S^{1} \rightarrow \mathcal{M}\right)$ as a graded manifold.

5. An independent issue is whether $\mathcal{Z}_{\bar{D}-\bar{Q}}$ is the ring of functions on an infinitedimensional (graded) manifold. This is linked to properties of the anchor map of the corresponding $L_{\infty}$-algebroid. ${ }^{2}$ It is easy, however, to use (1.12) and physics intuition to pull an appropriate manifold out of a hat for specific choices of $\mathcal{M}, \mathcal{N}, D$, and $Q$. When $\mathcal{Z}_{\bar{D}-\bar{Q}}$ is a graded manifold, the charts outlined in the previous remark should lead to an explicit atlas for it.

\subsection{Examples}

Real source Q-manifold examples. The main class of examples that we pull out of hats, and those that we discuss in detail, involve branes relevant to string/M-theory. The relevant source Q-manifold $\mathcal{N}$ is the shifted tangent bundle

$$
\mathcal{N}=T[1] \Sigma, \quad D=d,
$$

\footnotetext{
${ }^{1}$ We benefited from the discussion in [31] which is in a similar context to this paper.

${ }^{2} \mathrm{QP}$-manifolds and $L_{\infty}$-algebroids are identical notions (see e.g. [28]), possibly modulo technical details such as presence of absence of negative degree generators in the structure sheaf. The anchor of the $L_{\infty}$ algebroid is given via the derived bracket $(Q f, \bullet)$ for $f$ a function on the underlying manifold.
} 


\begin{tabular}{|c|c|c|}
\hline Brane & QP manifold $\mathcal{M}$ & Gen. Tangent Bundle \\
\hline F1 & $T^{\star}[2] T[1] M$ & $T \oplus T^{\star}$ \\
M2 & $T^{\star}[3] T[1] M$ & $T \oplus \Lambda^{2} T^{\star}$ \\
D3 & $T^{\star}[4] T[1] M \times \mathbb{R}^{2}[2]$ & $T \oplus T^{\star} \oplus T^{\star} \oplus \Lambda^{3} T^{\star}$ \\
M5 & $T^{\star}[6] T[1] M \times \mathbb{R}[3]$ & $T \oplus \Lambda^{2} T^{\star} \oplus \Lambda^{5} T^{\star}$ \\
\hline
\end{tabular}

Table 1. $p$-branes and their associated generalised geometries. All bundles have base $M$. (The source Q-manifold is $\mathcal{N}=T[1] \Sigma$ where the $p$-dimensional manifold $\Sigma$ is a spatial section of the worldvolume.)

where $\Sigma$ is a spatial slice of a brane worldvolume (e.g. of the form $\mathbb{R} \times \Sigma$ where $\mathbb{R}$ is time). The ring of functions on this $\mathcal{N}$ consists of ordinary differential forms on the (real, boundaryless, orientable) manifold $\Sigma$, with $d$ the de Rham differential, and the usual integral for $\int_{\mathcal{N}}$. The degree $P$ of the symplectic form on the target $\mathrm{QP}$ manifold $\mathcal{M}$ determines $\operatorname{dim} \Sigma=p$ by

$$
p=P-1
$$

as we will see later. Therefore, a $p$-brane is associated to a target-space QP manifold with symplectic form of degree $P=p+1$.

The zero locus $\mathcal{Z}_{\bar{D}-\bar{Q}}$ in these physically-motivated examples has a degree-zero subspace that turns out to be identical to the usual phase space of the hamiltonian formulation of brane dynamics. Note however that we only consider the bosonic sector, and so this degree-zero subspace is the phase space of the bosonic fields.

We list the brane phase spaces that we discuss in detail in this paper in table 1 . We only discuss the branes whose associated QP manifolds have been explored before (by Roytenberg [32] for the F1, by Ikeda-Uchino [33] and Grützmann [34] for the M2, ${ }^{3}$ and by the author [10] for the D3 and M5) just to keep the paper brief; it is rather straightforward to find QP manifolds for most familiar p-branes (including e.g. the IIA branes D2, D4, NS5A [36]) at least for $p \leq 5$.

Further examples in this class that we do not discuss in this paper include certain $P=2$ /Courant algebroid ones. An application involving a heterotic Courant algebroid [37] - which is not covered by the results of Alekseev and Strobl [2] — will be discussed in [38]. The WZW current or Kac-Moody algebra is a special case of the construction of section 3.2.1, for $M=G$ (a group manifold) with $H$-flux given by the canonical 3 -form $\left(g^{-1} d g\right)^{3}$; since it is studied in detail in [2], we do not discuss it. We expect to find interesting higher-dimensional analogues of Kac-Moody algebras (such as those of Cederwall, Ferretti, Nilsson, and Westerberg [39]) by specialising the construction of subsection 3.2 to $M=G$ and specialising the $H$-flux to one given by a group cocycle.

It will also be interesting to make contact with the charge algebra of Jurčo, Schupp, and Vysoký [40], which appears to involve a different choice of Q-structure and anchor map to all of the examples considered here.

\footnotetext{
${ }^{3}$ The relation to M-theory was originally pointed out by Kök enyesi, Sinkovics, and Szabo [35].
} 
As a final remark: a very similar general relationship between QP manifolds and brane hamiltonian dynamics, involving real Q-manifolds $\mathcal{N}(1.13)$ and the specific choice $\mathcal{M}=T^{\star}[P] T[1] M$ of section 3.2 was outlined by Ševera ([27], section 5) (albeit with a different choice of $\operatorname{dim} \Sigma$; it is unclear to us how to make contact with that formalism).

Complex source Q-manifold examples. A different class of examples is given by [41, 42]

$$
\mathcal{N}_{\mathrm{CY}}=T^{0,1}[1] \Sigma, \quad D=\bar{\partial}, \quad \int_{\mathcal{N}} f=\int_{\Sigma} \Omega \wedge f
$$

where now $\Sigma$ is a Calabi-Yau manifold with holomorphic volume form $\Omega$ and Dolbeault operator $\bar{\partial}$. This choice can be paired with any valid choice of target $\mathcal{M}$ to produce a holomorphic version of any of the previous examples. We have not yet attempted to tabulate interesting examples that arise this way, but it is plausible we could make contact with recent examples of (higher) holomorphic current algebras appearing in the context of holomorphic twists [43].

\section{The universal bracket formula and the brane phase space construction}

We prove the bracket formula (1.12), and provide the details of the construction of the brane phase space as the zero locus $\mathcal{Z}_{\bar{D}-\bar{Q}}$.

The starting point is a degree -1 graded Poisson bracket

$$
[F, G]
$$

for $F, G$ functionals on $\operatorname{Maps}(\mathcal{N} \rightarrow \mathcal{M})$. (This is not a BV-style antibracket, but rather a Schouten bracket; see subsection (3.1).) For every test function $\epsilon \in C^{\infty}(\mathcal{N})$, the smeared current

$$
\langle f \mid \epsilon\rangle \equiv(-1)^{P} \int_{\mathcal{N}} \boldsymbol{f} \epsilon
$$

associated to a function $f \in C^{\infty}(\mathcal{M})$ on the target space $\mathcal{M}$ is such a functional, of degree

$$
\operatorname{deg}\langle f \mid \epsilon\rangle=f+\epsilon-(P-1)
$$

(Here and elsewhere we omit deg when it is obvious, especially in sign factors.) We use the AKSZ construction to define the bracket $[\bullet, \bullet]$ in appendix A. For our purposes we will only need to use the formula

$$
[\langle f \mid \epsilon\rangle,\langle g \mid \eta\rangle]=-(-1)^{(g+P) \epsilon}\langle(f, g) \mid \epsilon \eta\rangle
$$

whose more explicit form is

$$
[\langle f \mid \epsilon\rangle,\langle g \mid \eta\rangle](\varphi)=(-1)^{P+1+(g+P) \epsilon} \int_{\mathcal{N}} \varphi^{\star}((f, g)) \epsilon \eta .
$$

As before, $(\bullet, \bullet)$ is the degree $-P$ Poisson bracket on $\mathcal{M}$, while $\varphi$ denotes an element of $\operatorname{Maps}(\mathcal{N} \rightarrow \mathcal{M})$. (We could have written $(\boldsymbol{f}, \boldsymbol{g})$ instead of $\varphi^{\star}((f, g))$, like in (1.10).) 
The Q-structures $Q$ and $D$ on the target and source graded manifolds $\mathcal{M}$ and $\mathcal{N}$ respectively lift to Q-structures $\bar{Q}$ and $\bar{D}$ on $\operatorname{Maps}(\mathcal{N} \rightarrow \mathcal{M})$, which act on currents as

$$
\bar{Q}\langle f \mid \epsilon\rangle=\langle Q f \mid \epsilon\rangle
$$

and

$$
\bar{D}\langle f \mid \epsilon\rangle=(-1)^{P} \int_{\mathcal{N}}(D \boldsymbol{f}) \epsilon=(-1)^{P+f+1} \int_{\mathcal{N}} \boldsymbol{f} D \epsilon .
$$

We used integration by parts (1.6) in the last formula. By abusing the bra-ket current notation slightly, we can write

$$
\bar{D}\langle f \mid \epsilon\rangle=\langle D f \mid \epsilon\rangle=(-1)^{f+1}\langle f \mid D \epsilon\rangle,
$$

The Q-structures $\bar{D}$ and $\bar{Q}$ mutually anticommute, so

$$
Z \equiv \bar{D}-\bar{Q}
$$

is a Q-structure:

$$
Z^{2}=0
$$

It is moreover compatible with the Poisson structure $[\bullet, \bullet]$ :

$$
Z[F, G]=[Z F, G]+(-1)^{F+1}[F, Z G]
$$

because $\bar{Q}$ and $\bar{D}$ are. Altogether, we have a graded Poisson algebra over $C^{\infty}(\operatorname{Maps}(\mathcal{N} \rightarrow$ $\mathcal{M})$ ), with degree -1 Poisson bracket $[\bullet, \bullet]$ and compatible Q-structure $Z$.

The zero-locus reduction of Grigoriev, Semikhatov, and Tipunin [9], takes the data of a graded Poisson algebra over $\mathcal{P}$ with compatible Q-structure $Z$ and degree $-n$ Poisson bracket $[\bullet, \bullet]$ and produces a graded Poisson algebra over the quotient $\mathcal{P} / \mathcal{I}_{Z}$ (where $\mathcal{I}_{Z}$ is the ideal generated by $Z$-exact elements) with degree $-(n-1)$ Poisson bracket

Proposition 2 (Zero-locus reduction [9]). $\mathcal{P} / \mathcal{I}_{Z}$ is a graded Poisson algebra with degree $-(n-1)$ Poisson bracket $\{\bullet, \bullet\}$ defined as the derived bracket

$$
\{\tilde{F}, \tilde{G}\} \equiv \widetilde{[F, Z(G)}], \quad F, G \in \mathcal{P},
$$

where $\tilde{F} \in \mathcal{P} / \mathcal{I}_{Z}$ is the equivalence class

$$
F \sim F+G Z(H), \quad F, G, H \in \mathcal{P} .
$$

Proof. Firstly, we calculate the degree of the new bracket:

$$
\operatorname{deg}\{\tilde{F}, \tilde{G}\}=\operatorname{deg} F+\operatorname{deg} G+\operatorname{deg} Z-n
$$

so $\operatorname{deg}\{\bullet, \bullet\}=-(n-1)$. 
The proof is a straightforward direct verification of the antisymmetry, Leibniz, and Jacobi identities for the new graded Poisson bracket. ${ }^{4}$ We display the calculation for the Jacobi identity only. We need to prove

$$
\{\tilde{F},\{\tilde{G}, \tilde{H}\}\}=\{\{\tilde{F}, \tilde{G}\}, \tilde{H}\}+(-1)^{(\tilde{F}+n+1)(\tilde{G}+n+1)}\{\tilde{G},\{\tilde{F}, \tilde{H}\}\} .
$$

We first write $X_{F} \equiv[F, \bullet]$ for the hamiltonian vector field (in the original bracket) associated to $F \in \mathcal{P}$, which satisfies

$$
\left[X_{F}, X_{G}\right] \equiv X_{F} X_{G}-(-1)^{X_{F} X_{G}} X_{G} X_{F}=X_{[F, G]}
$$

The calculation proving the Jacobi identity is below. We have unceremoniously omitted tildes and Z-exact terms. The tricky steps are the use of $Z^{2}=0$ and the compatibility condition

$$
Z[F, G]=[Z F, G]+(-1)^{F+n}[F, Z G]
$$

in the second and fourth lines, and the original bracket Jacobi identity in the fourth line.

$$
\begin{aligned}
\{F,\{G, H\}\} & \sim X_{F} Z X_{G} Z H \sim(-1)^{1+(G+n)(H+1+n)} X_{F} Z X_{Z H} G \\
& \sim(-1)^{1+(G+n+1)(H+1+n)} X_{F} X_{Z H} Z G \\
& \sim(-1)^{1+(G+n+1)(H+1+n)}\left[X_{F}, X_{Z H} Z G+(-1)^{1+(F+G+1)(H+1+n)} X_{Z H} X_{F} Z G\right. \\
& \sim(-1)^{1+(G+n+1)(H+1+n)} X_{[F, Z H]} Z G+(-1)^{1+(F+G)(H+1+n)}\{H,\{F, G\}\} \\
& \sim(-1)^{1+(G+n+1)(H+1+n)}\{\{F, H\}, G\}+\{\{F, G\}, H\} .
\end{aligned}
$$

We can rearrange the last line to $(-1)^{(F+n+1)(G+n+1)}\{G,\{F, H\}\}+\{\{F, G\}, H\}$ via antisymmetry of the new bracket, which concludes the proof of the Jacobi identity.

We arrive at the bracket formula (1.12) for the current algebra by simply applying the ZL reduction for $\mathcal{P}=C^{\infty}(\operatorname{Maps}(\mathcal{N} \rightarrow \mathcal{M})), Z=\bar{D}-\bar{Q}$, and $[\bullet, \bullet]$ the degree -1 bracket of (2.4). It remains to evaluate the bracket explicitly via (2.8), (2.6), and (2.4):

$$
\begin{aligned}
\widetilde{\langle f \mid \epsilon\rangle}, \widetilde{\langle f \mid \epsilon\rangle}\} & =\widehat{[\langle f \mid \epsilon\rangle,(\bar{D}-\bar{Q})\langle g \mid \eta\rangle}] \\
& =\widehat{\left[\langle f \mid \epsilon\rangle,(-1)^{g+1}\langle g \mid D \eta\rangle\right]}-\widehat{[\langle f \mid \epsilon\rangle,\langle Q g \mid \eta\rangle]} \\
& =(-1)^{(g+P) \epsilon+g} \widehat{\langle(f, g) \mid \epsilon D \eta\rangle}+(-1)^{(g+1+P) \epsilon} \overline{\langle(f, Q g) \mid \epsilon \eta\rangle} .
\end{aligned}
$$

This concludes the proof of the universal bracket formula (1.12).

\subsection{On perversion}

What if, instead of $Z=\bar{D}-\bar{Q}$, we had made a "perverse" choice

$$
Z=\alpha \bar{D}+\beta \bar{Q}, \quad \alpha, \beta \in \mathbb{R}
$$

\footnotetext{
${ }^{4}$ We follow the convention of [10] for these identities. There the Poisson bracket on target space $\mathcal{M}$ has degree $-p$, where $p$ is the degree of the associated symplectic form, which in this paper is denoted by $P$. For example, the Jacobi identity (2.15) matches the Jacobi identity of [10] for $p=n-1$.
} 
in the ZL reduction? This appears to adjust the relative coefficient of the Schwinger term in the current algebra (1.12), which is disturbing. Fortunately this freedom is illusory, outside of the case $\beta=0$ which must be excluded.

We consider the possible cases:

1. $\alpha \neq 0, \beta \neq 0$ : we can write $Z=\alpha(\bar{D}+(\beta / \alpha) \bar{Q})$, and rescale the Q-structure $Q$ on the target $\mathcal{M}$ to write this as $Z=\alpha(\bar{D}-\bar{Q})$. This is the case $Z=\bar{D}-\bar{Q}$, except the Poisson bivector has been rescaled by $\alpha$.

2. $\alpha=0$ : in this case we can again rescale $Q$ to set $\beta=-1$ and find

$$
\{\langle f \mid \epsilon\rangle,\langle g \mid \eta\rangle\}=(-1)^{\epsilon(g+P+1)}\langle(f, Q g) \mid \epsilon \eta\rangle .
$$

This says that all brackets close without Schwinger term, which is unexpected. However, in this case alone, most of the currents (1.9) turn out to vanish identically after the ZL quotient. For example, if we reinstate the tilde notation for the ZL quotient, the would-be Alexeev-Strobl current (3.35) becomes

$$
\widetilde{\langle A \mid \epsilon\rangle}=\widetilde{\int_{S^{1}}\left(v^{\mu}(\boldsymbol{x}) \chi_{\mu}+\alpha_{\mu}(\boldsymbol{x}) \boldsymbol{\psi}^{\mu}\right) \epsilon}=\overline{\int_{S^{1}} v^{\mu}(\boldsymbol{x}) \chi_{\mu} \epsilon} .
$$

The surviving currents are precisely the ones which have no Schwinger term in their current algebra. In other words, while the current algebra is sensible even if $\alpha=0$, we can no longer work with the convenient currents of the form (1.9).

3. $\beta=0$ : this case yields a different Poisson bracket structure to the other ones. This Poisson bracket will always be highly degenerate, in contrast to $\beta \neq 0$ which will be non-degenerate for reasonable target QP-manifolds $(\mathcal{M}, \omega, Q)$, except possibly for a finite-dimensional space of zero-modes as we shall see shortly.

\section{Examples of the brane phase space construction}

\subsection{Degree $P=1 \mathrm{QP}$ manifolds and the Schouten bracket}

Consider a QP manifold $\mathcal{M}$ with degree 1 symplectic form. It has Darboux coordinates

$$
\begin{array}{c|c|c}
\text { coord } & x^{\mu} & \xi_{\mu} \\
\operatorname{deg} & 0 & 1
\end{array}
$$

with $\left(x^{\mu}, \xi_{\nu}\right)=\delta_{\nu}^{\mu}$ and Q-structure arising from a hamiltonian

$$
\Theta=\frac{1}{2} \xi_{\mu} \pi^{\mu \nu}(x) \xi_{\nu}
$$

In this subsection alone the Grassmann parity will not equal the degree modulo two, so that some $x^{\mu}$ will be anticommuting. If $\operatorname{par} \xi_{\mu}=1+\operatorname{par} x^{\mu}$, we can identify the canonical Poisson bracket $(\bullet, \bullet)$ (of degree -1 ) with a (super) Schouten bracket, and $\pi^{\mu \nu}$ with a (super) Poisson tensor. Therefore the data of a QP manifold on $\mathcal{M}$ determines a super Poisson 
structure on the supermanifold $M$ with local coordinates $x^{\mu}$. It is not difficult to deduce that $\mathcal{M}$ is globally the shifted cotangent bundle $\mathcal{M}=T^{\star}[1] M$ (see [32], Proposition 3.1 for the purely bosonic case).

Per the general pattern, the current algebra construction requires a Q-manifold $\mathcal{N}$ with non-degenerate measure of degree $1-P=0$. The simplest choice is the 0 -dimensional manifold

$$
\mathcal{N}=\{\text { point }\}, \quad D=0,
$$

for which $C^{\infty}(\mathcal{N}) \cong \mathbb{R}$, and the measure is $\int_{\mathcal{N}} \epsilon=\epsilon$. We then find

$$
\operatorname{Maps}(\mathcal{N} \rightarrow \mathcal{M}) \cong \mathcal{M}
$$

so we identify the Poisson bracket $[\bullet, \bullet]$ on "functionals" with the Schouten bracket $(\bullet, \bullet)$.

The vector field $Z$ of the $Z L$ reduction is $Z=-Q$. Its zero locus ideal $\mathcal{I}_{Z}$ is generated by

$$
Q x^{\mu}=-\xi_{\nu} \pi^{\nu \mu}, \quad Q \xi_{\mu}=\frac{(-1)^{\mu(\rho+1)}}{2} \xi_{\nu} \partial_{\mu} \pi^{\nu \rho} \xi_{\rho} \quad\left((-1)^{\mu} \equiv(-1)^{\operatorname{par} x^{\mu}}\right) .
$$

When $\pi$ is non-degenerate, the zero locus $\mathcal{I}_{Z}$ is equivalent to the locus $\xi_{\mu}=0$; otherwise, $\xi_{\mu}=0$ defines a submanifold thereof, coisotropic under the Poisson bracket. The ZL reduction also works when the quotient is taken with respect to such a coisotropic submanifold [9]. If $f, g \in C^{\infty}(M)$, the expression

$$
\left.\{f, g\} \equiv(f,-Q g)\right|_{\xi=0}=\frac{\partial^{\text {right }} f}{\partial x^{\nu}} \pi^{\nu \mu} \frac{\partial g}{\partial x^{\mu}}
$$

is the new Poisson bracket produced by the ZL reduction. $\left(C^{\infty}(M)\right.$ is a geometrically convenient choice of representatives of the equivalence classes in the quotient.)

Since the currents (1.10) are in this case essentially identical to functions $f \in C^{\infty}(M)$, we see that for degree $P=1 \mathrm{QP}$ manifolds the current algebra construction produces a (super) Poisson bracket from the data of a Schouten bracket and a Poisson tensor (encoded as the self-commuting hamiltonian of the form (3.1)).

We end this somewhat degenerate example with the following observation: we saw previously that the general current algebra construction starts from a degree -1 graded Poisson bracket $[\bullet, \bullet]$ on $\operatorname{Maps}(\mathcal{N} \rightarrow \mathcal{M})$. This can be interpreted as a Schouten bracket. However the hamiltonian associated to $Z$ will in general not take the form (3.1), because there will exist coordinates with degrees $\neq 0,1$. Therefore, the general current algebra construction is not a special case of the degree $P=1$ construction discussed in this subsection, although it could perhaps be interpreted as the first step in a collection of "higher Poisson brackets" associated to a Schouten bracket plus a self-commuting hamiltonian ([44], Example 4.3).

\subsection{Generic $p$-brane without worldvolume gauge fields}

Here we again assume $M$ is an ordinary (real) manifold. For $P \geq 2$ we take the target QP manifold

$$
\mathcal{M}=T^{\star}[P] T[1] M
$$


with local homogeneous (in degree) coordinates

$$
\begin{array}{c|c|c|c|c|}
\operatorname{coord} & x^{\mu} & \psi^{\mu} & \chi_{\mu} & p_{\mu} \\
\operatorname{deg} & 0 & 1 & P-1 & P
\end{array}
$$

and nonvanishing Poisson brackets

$$
\left(x^{\mu}, p_{\nu}\right)=-\left(p_{\nu}, x^{\mu}\right)=\delta_{\nu}^{\mu}, \quad\left(\psi^{\mu}, \chi_{\nu}\right)=(-1)^{P}\left(\chi_{\nu}, \psi^{\mu}\right)=\delta_{\nu}^{\mu} .
$$

The target space Q-structure $Q$ has the hamiltonian ${ }^{5}$

$$
\Theta=-\psi^{\mu} p_{\mu}+\frac{1}{(P+1) !} H_{\mu_{1} \mu_{2} \cdots \mu_{P+1}}(x) \psi^{\mu_{1}} \psi^{\mu_{2}} \cdots \psi^{\mu_{P+1}}
$$

and $Q$ squares to zero when the $(P+1)$-form $H$ is closed. This form will turn out to couple electrically to the brane.

For the source Q-manifold $\mathcal{N}$ we take

$$
\mathcal{N}=T[1] \Sigma, \quad D=d,
$$

and for the measure $\int_{\mathcal{N}}$ we take the usual integral of differential forms on the (ordinary) manifold $\Sigma$. This measure has degree $1-P$ when $\operatorname{dim} \Sigma=P-1$. Hence $P$ is the worldvolume dimension (including time), and $p=P-1$. We normalise this measure as

$$
\int_{T[1] \Sigma} \frac{1}{p !} \epsilon_{\alpha_{1} \alpha_{2} \cdots \alpha_{p}}(\sigma) d \sigma^{\alpha_{1}} d \sigma^{\alpha_{2}} \cdots d \sigma^{\alpha_{p}} \equiv \int_{\Sigma} d^{p} \sigma \varepsilon^{\alpha_{1} \alpha_{2} \cdots \alpha_{p}} \frac{1}{p !} \epsilon_{\alpha_{1} \alpha_{2} \cdots \alpha_{p}}(\sigma)
$$

where the invariant Levi-Civita tensor density has $\varepsilon^{12 \cdots p}=1$.

An element $\varphi \in \operatorname{Maps}(\mathcal{N} \rightarrow \mathcal{M})$ is specified in local coordinates by the $4 \times(\operatorname{dim} M)$ superfields

$$
\varphi^{\star} x^{\mu}=\boldsymbol{x}^{\mu}, \quad \varphi^{\star} \psi^{\mu}=\boldsymbol{\psi}^{\mu}, \quad \varphi^{\star} \chi_{\mu}=\chi_{\mu}, \quad \varphi^{\star} p_{\mu}=\boldsymbol{p}_{\mu} .
$$

These are expanded in local coordinates $\sigma^{\alpha}, d \sigma^{\alpha}$ on $T[1] \Sigma$ (of degrees 0 and 1 respectively) to define component fields, e.g.

$$
\begin{aligned}
& \boldsymbol{x}^{\mu}=\sum_{n=0}^{p} \frac{1}{n !} x_{\alpha_{1} \alpha_{2} \cdots \alpha_{n}}^{\mu}(\sigma) d \sigma^{\alpha_{1}} d \sigma^{\alpha_{2}} \cdots d \sigma^{\alpha_{n}} \Longrightarrow \operatorname{deg} x_{\alpha_{1} \alpha_{2} \cdots \alpha_{n}}^{\mu}=-n, \\
& \chi_{\mu}=\sum_{n=0}^{p} \frac{1}{n !} \chi_{\mu \alpha_{1} \alpha_{2} \cdots \alpha_{n}}(\sigma) d \sigma^{\alpha_{1}} d \sigma^{\alpha_{2}} \cdots d \sigma^{\alpha_{n}} \Longrightarrow \operatorname{deg} \chi_{\mu \alpha_{1} \alpha_{2} \cdots \alpha_{n}}=p-n,
\end{aligned}
$$

with similar expansions for $\boldsymbol{\psi}^{\mu}$ and $\boldsymbol{p}_{\mu}$. Both superfields therefore contain component fields with vanishing intrinsic degree:

$$
\left.\boldsymbol{x}^{\mu}\right|_{\operatorname{deg} 0}=x^{\mu}(\sigma),\left.\quad \chi_{\mu}\right|_{\operatorname{deg} 0}=\frac{1}{p !} \chi_{\mu \alpha_{1} \cdots \alpha_{p}}(\sigma) d \sigma^{\alpha_{1}} \cdots d \sigma^{\alpha_{p}} .
$$

\footnotetext{
${ }^{5}$ Potential $\psi^{2} \chi$ terms can be removed by a symplectomorphism. See ([10], section 2.3).
} 
The ZL reduction with respect to $Z \equiv \bar{d}-\bar{Q}$ eliminates $\boldsymbol{\psi}^{\mu}$ and $\boldsymbol{p}_{\mu}$ in favour of $\boldsymbol{x}^{\mu}$ and $\chi_{\mu}$ :

$$
d \boldsymbol{x}^{\mu}=\boldsymbol{\psi}^{\mu}, \quad d \boldsymbol{\chi}_{\mu}=(-1)^{p} \boldsymbol{p}_{\mu}+\frac{1}{P !} H_{\nu_{1} \nu_{2} \cdots \nu_{P} \mu}(\boldsymbol{x}) \boldsymbol{\psi}^{\nu_{1}} \boldsymbol{\psi}^{\nu_{2}} \cdots \boldsymbol{\psi}^{\nu_{P}}
$$

(The remaining ZL conditions do not imply further constraints.) Therefore, to determine the Poisson brackets between all component fields, we only need consider the currents $\left\langle x^{\mu}\right|$ and $\left\langle\chi_{\mu}\right|$. The choice of "test" function $\epsilon \in C^{\infty}(T[1] \Sigma) \cong \Lambda^{\bullet}(\Sigma)$ determines which component field appears in the current. For $\epsilon=\epsilon_{\alpha_{1} \alpha_{2} \cdots \alpha_{p-n}}(\sigma) d \sigma^{\alpha_{1}} d \sigma^{\alpha_{2}} \cdots d \sigma^{\alpha_{p-n}} /(p-n)$ ! we pick up the $n$-form component:

$$
(-1)^{p+1}\left\langle x^{\mu} \mid \epsilon\right\rangle \equiv \int_{T[1] \Sigma} \boldsymbol{x}^{\mu} \epsilon=\frac{p !}{n !(p-n) !} \int_{\Sigma} d^{p} \sigma \varepsilon^{\alpha_{1} \alpha_{2} \cdots \alpha_{p}} \frac{1}{p !} x_{\alpha_{1} \alpha_{2} \cdots \alpha_{n}}^{\mu}(\sigma) \epsilon_{\alpha_{n+1} \cdots \alpha_{p}}(\sigma) .
$$

Poisson brackets. We calculate the Poisson brackets of component fields via the universal bracket formula (1.12), that gives

$$
\left\{\left\langle x^{\mu} \mid \epsilon_{1}\right\rangle,\left\langle\chi_{\nu} \mid \epsilon_{2}\right\rangle\right\}=(-1)^{p}\left\langle\delta_{\nu}^{\mu} \mid \epsilon_{1} \epsilon_{2}\right\rangle=-\delta_{\nu}^{\mu} \int_{T[1] \Sigma} \epsilon_{1} \epsilon_{2}
$$

Evidently this pairs $n$-form with $(p-n)$-form components. To display the brackets of component fields, we rewrite the $\chi_{\mu}$ component fields as the tensor densities

$$
P_{\mu}^{\alpha_{1} \alpha_{2} \cdots \alpha_{n}} \equiv-\frac{1}{(p-n) !} \varepsilon^{\beta_{1} \beta_{2} \cdots \beta_{p-n} \alpha_{1} \cdots \alpha_{n}} \chi_{\mu \beta_{1} \beta_{2} \cdots \beta_{p-n}} .
$$

Then (3.16) gives the Poisson brackets

$$
\left\{x_{\alpha_{1} \alpha_{2} \cdots \alpha_{n}}^{\mu}\left(\sigma_{1}\right), P_{\nu}^{\beta_{1} \beta_{2} \cdots \beta_{m}}\left(\sigma_{2}\right)\right\}=n !(-1)^{n(p-n)} \delta_{m n} \delta_{\nu}^{\mu} \delta_{\alpha_{1} \alpha_{2} \cdots \alpha_{n}}^{\beta_{1} \beta_{2} \cdots \beta_{m}} \delta^{p}\left(\sigma_{1}-\sigma_{2}\right),
$$

where $0 \leq n \leq p$. Similarly, $\left\{\left\langle x^{\mu} \mid \epsilon_{1}\right\rangle,\left\langle x^{\nu} \mid \epsilon_{2}\right\rangle\right\}=0$ implies

$$
\left\{x_{\alpha_{1} \alpha_{2} \cdots \alpha_{n}}^{\mu}\left(\sigma_{1}\right), x_{\beta_{1} \beta_{2} \cdots \beta_{n}}^{\nu}\left(\sigma_{2}\right)\right\}=0 .
$$

The last bracket, $\left\{P_{\mu}^{\alpha_{1} \alpha_{2} \cdots \alpha_{n}}\left(\sigma_{1}\right), P_{\nu}^{\beta_{1} \beta_{2} \cdots \beta_{m}}\left(\sigma_{2}\right)\right\}$, is fixed by

$$
\left\{\left\langle\chi_{\mu} \mid \epsilon_{1}\right\rangle,\left\langle\chi_{\nu} \mid \epsilon_{2}\right\rangle\right\}=\frac{(-1)^{p}}{p !}\left\langle H_{\mu \nu \rho_{1} \rho_{2} \cdots \rho_{p}}(x) \psi^{\rho_{1}} \psi^{\rho_{2}} \cdots \psi^{\rho_{p}} \mid \epsilon_{1} \epsilon_{2}\right\rangle .
$$

Unless $H=0$ the result is too horrific to calculate or display, except for $n=m=0$ :

$$
\left\{P_{\mu}\left(\sigma_{1}\right), P_{\nu}\left(\sigma_{2}\right)\right\}=-\frac{1}{p !} H_{\mu \nu \rho_{1} \rho_{2} \cdots \rho_{p}} \varepsilon^{\alpha_{1} \cdots \alpha_{p}} \partial_{\alpha_{1}} x^{\rho_{1}} \cdots \partial_{\alpha_{p}} x^{\rho_{p}} .
$$

We deduce therefore that the zero locus $\mathcal{Z}_{\bar{D}-\bar{Q}}$ in this case is the infinite-dimensional cotangent bundle $T^{\star} \operatorname{Maps}(T[1] \Sigma \rightarrow M)$, which is a manifold. The degree-zero component fields

$$
X^{\mu}(\sigma) \equiv x^{\mu}(\sigma), \quad P_{\mu}(\sigma)
$$


satisfy the Poisson bracket relations

$$
\begin{gathered}
\left\{X^{\mu}\left(\sigma_{1}\right), P_{\nu}\left(\sigma_{2}\right)\right\}=\delta_{\nu}^{\mu} \delta^{p}\left(\sigma_{1}-\sigma_{2}\right), \quad\left\{X^{\mu}\left(\sigma_{1}\right), X^{\nu}\left(\sigma_{2}\right)\right\}=0 . \\
\left\{P_{\mu}\left(\sigma_{1}\right), P_{\nu}\left(\sigma_{2}\right)\right\}=-\frac{1}{p !} H_{\mu \nu \rho_{1} \rho_{2} \cdots \rho_{p}} \varepsilon^{\alpha_{1} \cdots \alpha_{p}} \partial_{\alpha_{1}} X^{\rho_{1}} \cdots \partial_{\alpha_{p}} X^{\rho_{p}}
\end{gathered}
$$

For $H=0$ these are the canonical Poisson brackets of $p$-brane positions and momenta. Otherwise, they account for the contribution to the symplectic structure due to an electric coupling $\int_{\mathbb{R} \times \Sigma} H$ involving the background $(p+2)$-form $H$. This degree zero sector corresponds to the body of $T^{\star} \operatorname{Maps}(T[1] \Sigma \rightarrow M)$ - in the supermanifold sense - which is just the cotangent bundle of the higher loop space $\operatorname{Maps}(\Sigma \rightarrow M) . T^{\star} \operatorname{Maps}(\Sigma \rightarrow M)$ is the usual $p$-brane phase space for a worldvolume $\mathbb{R} \times \Sigma$ (in the absence of worldvolume gauge fields).

Current algebra. We consider the current algebra of the zero-degree currents of the form (1.10). The top form ( $p$-form) currents $\langle A|$ arise from functions $A$ of degree $\operatorname{deg} A=$ $P-1=p$ on $\mathcal{M}$, which correspond to sections $(v, \alpha)$ of $T M \oplus \Lambda^{p} T^{\star} M$ :

$$
A=-v^{\mu}(x) \chi_{\mu}+\frac{1}{p !} \alpha_{\mu_{1} \cdots \mu_{p}}(x) \psi^{\mu_{1}} \cdots \psi^{\mu_{p}} .
$$

The corresponding current, smeared against a 0-form $\epsilon=\epsilon(\sigma)$, takes the form

$$
\langle A \mid \epsilon\rangle=(-1)^{p+1} \int_{T[1] \Sigma}\left(-v^{\mu}(\boldsymbol{x}) \chi_{\mu}+\frac{1}{p !} \alpha_{\mu_{1} \cdots \mu_{p}}(\boldsymbol{x}) d \boldsymbol{x}^{\mu_{1}} \cdots d \boldsymbol{x}^{\mu_{p}}\right) \epsilon(\sigma)
$$

where we already imposed the ZL reduction (3.14) to express the current in terms of the independent superfields $\boldsymbol{x}^{\mu}(\sigma, d \sigma)$ and $\boldsymbol{\chi}_{\mu}(\sigma, d \sigma)$. The importance of the degree-zero currents is that they have a non-vanishing contribution even after we set the $\operatorname{deg} \neq 0$ component fields in $\boldsymbol{x}^{\mu}$ and $\boldsymbol{\chi}_{\mu}$ to zero (as in (3.13)): after resolving the $\int_{T[1] \Sigma}$ integral, we find

$$
\left.\langle A \mid \epsilon\rangle\right|_{\operatorname{deg} 0}=(-1)^{p+1} \int_{\Sigma} d^{p} \sigma\left(v^{\mu}(X) P_{\mu}+\frac{1}{p !} \varepsilon^{\alpha_{1} \cdots \alpha_{p}} \alpha_{\mu_{1} \cdots \mu_{p}}(X) \partial_{\alpha_{1}} X^{\mu_{1}} \cdots \partial_{\alpha_{p}} X^{\mu_{p}}\right) \epsilon(\sigma) .
$$

These currents and their current algebra were considered by Zabzine and Bonelli [11]. We will see shortly that they reduce to the Alekseev-Strobl currents for $p=1$.

If $(v, \alpha),(u, \beta)$ are sections of $T M \oplus \Lambda^{p} T^{\star} M$ defining the degree $p$ functions

$$
A=-v^{\mu}(x) \chi_{\mu}+\frac{1}{p !} \alpha_{\mu_{1} \cdots \mu_{p}}(x) \psi^{\mu_{1}} \cdots \psi^{\mu_{p}}, \quad B=-u^{\mu}(x) \chi_{\mu}+\frac{1}{p !} \beta_{\mu_{1} \cdots \mu_{p}}(x) \psi^{\mu_{1}} \cdots \psi^{\mu_{p}}
$$

we calculate the following expression that appears in the current algebra using the Poisson brackets (3.7)

$$
\begin{aligned}
(-1)^{p+1}(B, Q A)= & \left(v^{\nu} \partial_{\nu} u^{\mu}-u^{\nu} \partial_{\nu} v^{\mu}\right)\left(-\chi_{\mu}\right) \\
& +\left(v^{\nu} \partial_{\nu} \beta_{\mu_{1} \cdots \mu_{p}}+p \beta_{\nu \mu_{2} \cdots \mu_{p}} \partial_{\mu_{1}} v^{\nu}-(p+1) u^{\nu} \partial_{[\nu} \alpha_{\left.\mu_{1} \cdots \mu_{p}\right]}\right. \\
& \left.+(-1)^{p+1} v^{\nu} u^{\rho} H_{\nu \rho \mu_{1} \cdots \mu_{p}}\right) \frac{\psi^{\mu_{1}} \psi^{\mu_{2}} \cdots \psi^{\mu_{p}}}{p !}
\end{aligned}
$$


Notwithstanding the sign factor $(-1)^{p+1}$, this is the Vinogradov bracket ([45], Proposition 5.9), $[28]^{6}$

$$
(-1)^{p+1}[(v, \alpha),(u, \beta)]_{\mathrm{V}}=\left([v, u], \mathcal{L}_{v} \beta-\iota_{u} d \alpha+(-1)^{p+1} \iota_{v} \iota_{u} H\right) .
$$

We also need the following expression, which is also part of the definition of a Vinogradov algebroid:

$$
(A, B)=(-1)^{p}\left(u^{\mu} \alpha_{\mu \nu_{1} \cdots \mu_{p}}+v^{\mu} \beta_{\mu \nu_{1} \cdots \mu_{p-1}}\right) \frac{\psi^{\mu_{1}} \psi^{\mu_{2}} \cdots \psi^{\mu_{p-1}}}{(p-1) !}=(B, A) .
$$

Finally, the bracket formula (1.12) yields, for the case $\operatorname{deg} \epsilon=\operatorname{deg} \eta=0$ of interest,

$$
\{\langle A \mid \epsilon\rangle,\langle B \mid \eta\rangle\}=-\langle(B, Q A) \mid \eta \epsilon\rangle+(-1)^{p+1}\langle(A, B) \mid \eta d \epsilon\rangle
$$

where

$$
\begin{aligned}
-\langle(B, Q A) \mid \eta \epsilon\rangle & =-\int_{T[1] \Sigma}\left([v, u]^{\mu}\left(-\boldsymbol{\chi}_{\mu}\right)+\ldots\right) \eta \epsilon \\
(-1)^{p+1}\langle(A, B) \mid \eta d \epsilon\rangle & =\int_{T[1] \Sigma}(-1)^{p}\left(u^{\mu} \sigma_{\mu \nu_{1} \cdots \mu_{p}}+v^{\mu} \beta_{\mu \nu_{1} \cdots \mu_{p-1}}\right) \frac{d \boldsymbol{x}^{\mu_{1}} d \boldsymbol{x}^{\mu_{2}} \cdots d \boldsymbol{x}^{\mu_{p-1}}}{(p-1) !} \eta d \epsilon .
\end{aligned}
$$

By taking the degree-zero $\left.\right|_{\operatorname{deg} 0}$ parts of all superfields, we immediately recover the result of [11] for the currents (3.26). In other words, we have shown that the canonical $p$-brane Poisson brackets (3.23) generate a current algebra that is controlled by the geometry of the Vinogradov algebroid over $T M \oplus \Lambda^{p} T^{\star} M$. The term $\langle(A, B) \mid \eta d \epsilon\rangle$ is recognised as a Schwinger term when the $\int_{T[1] \Sigma}$ expression is resolved:

$$
\begin{aligned}
& \left.\langle(A, B) \mid \eta d \epsilon\rangle\right|_{\operatorname{deg} 0}= \\
& -\int_{\Sigma} d^{p} \sigma\left(u^{\mu} \alpha_{\mu \nu_{1} \cdots \mu_{p}}+v^{\mu} \beta_{\mu \nu_{1} \cdots \mu_{p-1}}\right) \frac{\varepsilon^{\alpha_{1} \cdots \alpha_{p-1} \beta}}{(p-1) !} \partial_{\alpha_{1}} X_{1}^{\mu} \cdots \partial_{\alpha_{p-1}} X^{\mu_{p-1}} \eta \partial_{\beta} \epsilon .
\end{aligned}
$$

There also exist further degree-zero currents (1.10), which are $n$-forms for $0 \leq n<p$, arising from functions of the form

$$
\lambda_{\mu_{1} \cdots \mu_{n}}(x) \psi^{\mu_{1}} \cdots \psi^{\mu_{n}} .
$$

For $n=p-1$ these appear in the Schwinger term $\langle(A, B) \mid \eta d \epsilon\rangle$. More generally, formula (1.12) implies that the Schwinger term in the Poisson bracket of an $n$-form and an $m$-form bracket will involve an $(n+m-p)$-form current.

\footnotetext{
${ }^{6}$ Sometimes "Vinogradov bracket" refers to the antisymmetrisation of this bracket.
} 


\subsubsection{F1 brane, or the Alekseev-Strobl current algebra $(p=1)$}

For $p=1$, the top-form currents (3.26) (corresponding to 1 -forms on the circle $S^{1}$ ) take the form

$$
\left.\langle A \mid \epsilon\rangle\right|_{\operatorname{deg} 0}=\int_{\Sigma} d^{1} \sigma\left(v^{\mu}(X) P_{\mu}+\alpha_{\mu}(X) \partial_{\sigma} X^{\mu}\right) \epsilon(\sigma)=\int_{\Sigma} d^{1} \sigma J(v, \alpha)(\sigma) \epsilon(\sigma) .
$$

These are the Alekseev-Strobl string currents [2]. The Poisson brackets of the degree-zero component fields $X^{\mu}(\sigma), P_{\nu}(\sigma)$ are defined by (3.23) for $p=1$ :

$$
\begin{aligned}
& \left\{X^{\mu}\left(\sigma_{1}\right), P_{\nu}\left(\sigma_{2}\right)\right\}=\delta_{\nu}^{\mu} \delta^{p}\left(\sigma_{1}-\sigma_{2}\right), \quad\left\{X^{\mu}\left(\sigma_{1}\right), X^{\nu}\left(\sigma_{2}\right)\right\}=0 . \\
& \left\{P_{\mu}\left(\sigma_{1}\right), P_{\nu}\left(\sigma_{2}\right)\right\}=-H_{\mu \nu \rho} \partial_{\sigma} X^{\rho} .
\end{aligned}
$$

Then formulas (3.31), (3.32), (3.28), and (3.30) determine the Poisson bracket $\{\langle A \mid \epsilon\rangle,\langle B \mid \eta\rangle\}$ of the smeared current (3.35) with another such current $\langle B \mid \eta\rangle=\int_{\Sigma} d^{1} \sigma J(u, \beta)(\sigma) \eta(\sigma)$. We recover therefore the Alekseev-Strobl current algebra formula (quoted in the introduction (1.5) for the special case of no H-flux).

For this case of $p=1$, the space $\operatorname{Maps}(T[1] \Sigma \rightarrow M)$ has been interpreted as a superspace for standard $N=1$ worldsheet supersymmetry by Zabzine [46]. For $p>1$ however a similar interpretation appears to be unavailable. Presumably this is related to the notorious difficulties in the construction of "spinning" or "RNS"-style membrane and higher brane lagrangians.

\subsubsection{M2 brane}

We point out for completeness that for $p=2$, we have $\operatorname{dim} \Sigma=2$, and the degree-zero subspace of the zero locus is the M2 brane phase space $T^{\star} \operatorname{Maps}(\Sigma \rightarrow M)$, with a symplectic structure that will in general depend on the 4-form flux $H$.

\subsection{M5 brane}

Here the source $\mathrm{Q}$ manifold is as in the previous case, $\mathcal{N}=T[1] \Sigma$, but with $\operatorname{dim} \Sigma=5$, while the target QP manifold is:

$$
\mathcal{M}=T^{\star}[6] T[1] M \times \mathbb{R}[3]
$$

with local homogeneous (in degree) coordinates

$$
\begin{array}{c|c|c|c|c|c|}
\operatorname{coord} & x^{\mu} & \psi^{\mu} & \zeta & \chi_{\mu} & p_{\mu} \\
\operatorname{deg} & 0 & 1 & 3 & 5 & 6
\end{array}
$$

and nonvanishing Poisson brackets

$$
\left(x^{\mu}, p_{\nu}\right)=-\left(p_{\nu}, x^{\mu}\right)=\delta_{\nu}^{\mu}, \quad\left(\psi^{\mu}, \chi_{\nu}\right)=\left(\chi_{\nu}, \psi^{\mu}\right)=\delta_{\nu}^{\mu}, \quad(\zeta, \zeta)=1
$$

The target space Q-structure $Q$ has the hamiltonian

$$
\Theta=-\psi^{\mu} p_{\mu}+\frac{1}{7 !} F_{\mu_{1} \mu_{2} \cdots \mu_{7}}(x) \psi^{\mu_{1}} \psi^{\mu_{2}} \cdots \psi^{\mu_{7}}+\frac{1}{4 !} G_{\mu_{1} \mu_{2} \cdots \mu_{4}}(x) \psi^{\mu_{1}} \psi^{\mu_{2}} \cdots \psi^{\mu_{4}} \zeta
$$


and $Q$ squares to zero when

$$
d G=0, \quad d F+\frac{1}{2} G \wedge G=0 .
$$

The symplectic structure on $\mathcal{M}$ evidently has degree $P=6$, so that $p=5$. This $\mathrm{QP}$ structure was written down in [10], which matches the convention here under $\psi \rightarrow-\psi$, $\chi \rightarrow-\chi$.

The novel ingredient compared to section 3.2 is the coordinate $\zeta$ along $\mathbb{R}[3]$ of degree 3 . The corresponding superfield is

$$
\boldsymbol{\zeta}(\sigma, d \sigma)=\sum_{n=0}^{5} \frac{1}{n !} \zeta_{\alpha_{1} \cdots \alpha_{n}}(\sigma) d \sigma^{\alpha_{1}} \cdots d \sigma^{\alpha_{n}} \Longrightarrow \operatorname{deg} \zeta_{\alpha_{1} \cdots \alpha_{n}}=3-n .
$$

It is also convenient to work with the dualised component fields

$$
\zeta^{\alpha_{1} \cdots \alpha_{n}}=\frac{1}{(5-n) !} \varepsilon^{\alpha_{1} \cdots \alpha_{n} \beta_{1} \cdots \beta_{5-n}} \zeta_{\beta_{1} \cdots \beta_{5-n}} \Longrightarrow \operatorname{deg} \zeta^{\alpha_{1} \cdots \alpha_{n}}=n-2
$$

Formula (1.12) yields their mutual brackets: since $\{\langle\zeta \mid \epsilon\rangle,\langle\zeta \mid \eta\rangle\}=(-1)^{\epsilon+1}\langle 1 \mid \epsilon d \eta\rangle$, we find

$$
\left\{\zeta^{\alpha_{1} \cdots \alpha_{n}}\left(\sigma_{1}\right), \zeta^{\beta_{1} \cdots \beta_{m}}\left(\sigma_{2}\right)\right\}=-\varepsilon^{\gamma \alpha_{1} \cdots \alpha_{n} \beta_{1} \cdots \beta_{m}} \frac{\partial}{\partial \sigma_{1}^{\gamma}} \delta^{5}\left(\sigma_{1}-\sigma_{2}\right) \delta_{m, 4-n} .
$$

To discuss the current algebra and ZL reduction it is convenient to first switch off the 4-form and 7-form background fluxes $G$ and $F$ :

Poisson brackets for vanishing fluxes. In this case

$$
\Theta=-\psi^{\mu} p_{\mu}
$$

The ZL reduction reads

$$
d \boldsymbol{x}^{\mu}=\boldsymbol{\psi}^{\mu}, \quad d \boldsymbol{\chi}_{\mu}=-\boldsymbol{p}_{\mu}, \quad d \boldsymbol{\zeta}=0
$$

The independent superfields are $\boldsymbol{x}^{\mu}, \boldsymbol{\chi}_{\mu}$ and $\boldsymbol{\zeta}$, where the latter is a closed polyform:

$$
d \zeta=0 \Longleftrightarrow \partial_{\left[\alpha_{1}\right.} \zeta_{\left.\alpha_{2} \cdots \alpha_{n+1}\right]}=0 \Longleftrightarrow \partial_{\alpha} \zeta^{\alpha \beta_{1} \cdots \beta_{n}}=0 \text {. }
$$

The non-vanishing Poisson brackets of the component fields are

$$
\begin{aligned}
\left\{x_{\alpha_{1} \alpha_{2} \cdots \alpha_{n}}^{\mu}\left(\sigma_{1}\right), P_{\nu}^{\beta_{1} \beta_{2} \cdots \beta_{m}}\left(\sigma_{2}\right)\right\} & =n !(-1)^{n(p-n)} \delta_{m n} \delta_{\nu}^{\mu} \delta_{\alpha_{1} \alpha_{2} \cdots \alpha_{n}}^{\beta_{1} \beta_{2} \cdots \beta_{m}} \delta^{5}\left(\sigma_{1}-\sigma_{2}\right), \\
\left\{\zeta^{\alpha_{1} \cdots \alpha_{n}}\left(\sigma_{1}\right), \zeta^{\beta_{1} \cdots \beta_{m}}\left(\sigma_{2}\right)\right\} & =-\varepsilon^{\gamma \alpha_{1} \cdots \alpha_{n} \beta_{1} \cdots \beta_{m}} \frac{\partial}{\partial \sigma_{1}^{\gamma}} \delta^{5}\left(\sigma_{1}-\sigma_{2}\right) \delta_{m, 4-n}
\end{aligned}
$$

where $P_{\mu}^{\alpha_{1} \cdots \alpha_{n}}$ was defined in terms of $\boldsymbol{\chi}_{\mu}$ in (3.17), and the component fields for $\boldsymbol{x}$ and $\boldsymbol{\zeta}$ in (3.12) and (3.42) respectively. 
This Poisson structure is degenerate. To see this, select a Riemannian metric on $\Sigma$ (perhaps the one induced by a Lorentzian metric on the worldvolume $\mathbb{R} \times \Sigma$ ) to produce a Hodge decomposition:

$$
C^{\infty}(T[1] \Sigma) \cong \Lambda^{\bullet}(\Sigma)=E \oplus C \oplus H
$$

where $E, C$, and $H$ are $d$-exact, co-exact, and harmonic forms respectively. $\Lambda^{\bullet}(\Sigma)$ is a (graded) symplectic vector space with non-degenerate (graded) symplectic form $\omega$

$$
\omega(\epsilon, \eta) \equiv \int_{T[1] \Sigma} \epsilon \eta
$$

and the harmonic forms are symplectically-orthogonal to the rest:

$$
\omega(H, E)=\omega(H, C)=0 .
$$

Therefore the current $\langle\zeta \mid \eta\rangle$ depends only on the harmonic part of the superfield $\zeta$ if $\eta$ is selected to be harmonic. However, when that is the case, we calculate

$$
\{\langle\zeta \mid \epsilon\rangle,\langle\zeta \mid \eta\rangle\}=(-1)^{\epsilon+1}\langle 1 \mid \epsilon d \eta\rangle=0 \quad \forall \epsilon \in C^{\infty}(T[1] \Sigma)
$$

since $d \eta=0$ for all harmonic forms $\eta$. Thus, the harmonic part of the superfield $\boldsymbol{\zeta}$ drops out of all Poisson brackets.

Since $\omega$ is non-degenerate on the subspace of exact and co-exact forms $E \oplus C$, we deduce similarly that the Poisson structure (3.47) is non-degenerate on exact $\boldsymbol{\zeta}$.

M5-brane phase space and self-duality. Let us make contact with the hamiltonian formulation of the bosonic M5 brane [47]. The canonical variables are the brane position $X^{\mu}(\sigma)$, the momentum density $P_{\nu}(\sigma)$, the spatial component $A_{\alpha_{1} \alpha_{2}}(\sigma)$ of the 2 -form gauge field on the worldvolume, and its conjugate momentum $\Pi^{\alpha_{1} \alpha_{2}}(\sigma)$. The lagrangian density is

$$
P_{\mu} \dot{X}^{\mu}+\frac{1}{2} \Pi^{\alpha_{1} \alpha_{2}} \dot{A}_{\alpha_{1} \alpha_{2}}-\lambda_{\alpha_{1} \alpha_{2}}\left(\Pi^{\alpha_{1} \alpha_{2}}-T \varepsilon^{\alpha_{1} \alpha_{2} \beta_{1} \beta_{2} \beta_{3}} \partial_{\beta_{1}} A_{\beta_{2} \beta_{3}}\right)+\ldots
$$

where $\lambda_{\alpha_{1} \alpha_{2}}$ is a lagrange multiplier, $T$ is the brane tension, and we omitted the worldvolume diffeomorphism constraints and their lagrange multipliers.

The expression

$$
\Pi^{\alpha_{1} \alpha_{2}}-T \varepsilon^{\alpha_{1} \alpha_{2} \beta_{1} \beta_{2} \beta_{3}} \partial_{\beta_{1}} A_{\beta_{2} \beta_{3}}=0
$$

defines a mix of second and first-class constraints, of which the latter imply the Gauss law for this 2-form electrodynamics [48]. Passing to (partial) Dirac brackets yields

$$
\left\{\Pi^{\alpha_{1} \alpha_{2}}\left(\sigma_{1}\right), \Pi^{\beta_{1} \beta_{2}}\left(\sigma_{2}\right)\right\}_{\text {Dirac }} \propto-\varepsilon^{\gamma \alpha_{1} \alpha_{2} \beta_{1} \beta_{2}} \frac{\partial}{\partial \sigma_{1}^{\gamma}} \delta^{5}\left(\sigma_{1}-\sigma_{2}\right) .
$$

This is identical to the bracket (3.47) for the degree zero component fields $\zeta^{\alpha_{1} \alpha_{2}}$. Since the bracket (3.47) is only non-degenerate when the component fields are $d$-exact, we should be writing

$$
\zeta_{\alpha_{1} \alpha_{2} \alpha_{3}} \propto \partial_{\left[\alpha_{1}\right.} A_{\left.\alpha_{2} \alpha_{3}\right]}
$$


for some 2-form potential $A_{\alpha_{1} \alpha_{2}}$. After dualisation, we find

$$
\zeta^{\alpha_{1} \alpha_{2}} \propto \varepsilon^{\alpha_{1} \alpha_{2} \beta_{1} \beta_{2} \beta_{3}} \partial_{\beta_{1}} A_{\beta_{2} \beta_{3}} .
$$

Therefore the current algebra construction for $\mathcal{M}=T^{\star}[6] T[1] M \times \mathbb{R}[3]$ contains the reduced bosonic M5-brane phase space, where the constraint (3.53) has been imposed.

That constraint is simply the self-duality of the gauge field on the M5-brane worldvolume, expressed in a time/space split. If $H$ is the 3 -form field strength on $\mathbb{R} \times \Sigma$ with $\mathbb{R}$ the time $t$ direction, $H$ will decompose as $H=\Pi d t+\zeta$, where $\zeta$ is a spatial 3 -form $\zeta \in \Lambda^{3}(\Sigma)$, and $\Pi$ is a 2 -form $\Pi \in \Lambda^{2}(\Sigma)$. Assuming for simplicity that the 6-dimensional worldvolume metric is block-diagonal, we see immediately that a self-duality condition of the form $\star_{6} H \propto H$ will produce $\star_{5} \zeta \propto \Pi$, which is the constraint (3.53) modulo index gymnastics. ${ }^{7}$

Current algebra for vanishing fluxes. The top-form currents correspond to $A \in$ $C^{\infty}(\mathcal{M})$ of degree $p=5$ :

$$
A=v^{\mu}(x)\left(-\chi_{\mu}\right)+\frac{1}{2} \omega_{\mu_{1} \mu_{2}}(x) \psi^{\mu_{1}} \psi^{\mu_{2}} \zeta+\frac{1}{5 !} \sigma_{\mu_{1} \cdots \mu_{5}}(x) \psi^{\mu_{1}} \cdots \psi^{\mu_{5}} .
$$

This is a section of the bundle $E$

$$
E=\left(T \oplus \Lambda^{2} T^{\star} \oplus \Lambda^{5} T^{\star}\right) M
$$

of generalised vectors for $E_{6(6)}$ exceptional generalised geometry (for $\operatorname{dim} M=6$ ) or

$$
E_{d(d)}=\operatorname{Spin}(5,5), \operatorname{SL}(5), \ldots
$$

(for $d=5,4, \ldots$ ), in an M-theory section $[49,50]$. Other dimensions $d$ are allowed in our construction, but the fibres of $E$ will fail to admit an action of the group $E_{d(d)}$. The geometric expressions $\left(A^{\prime}, Q A\right)$ and $\left(A^{\prime}, A\right)$ that enter the current algebra were explicitly calculated in [10]. They correspond to the generalised Lie derivative

$$
\left(A^{\prime}, Q A\right)=L_{A} A^{\prime}=\mathcal{L}_{v} v^{\prime}+\left(\mathcal{L}_{v} \omega^{\prime}-\imath_{v^{\prime}} d \omega\right)+\left(\mathcal{L}_{v} \sigma^{\prime}-\imath_{v^{\prime}} d \sigma-\omega^{\prime} \wedge d \omega\right)
$$

and the symmetric map $\times_{N}: E \otimes E \rightarrow N \equiv\left(T^{\star} \oplus \Lambda^{4} T^{\star}\right) M$ (sometimes denoted by $\bullet$ ) related to the symmetric part of the generalised Lie derivative ${ }^{8}$

$$
\begin{aligned}
\left(A, A^{\prime}\right) & =-\left(v^{\prime \mu} \omega_{\mu \nu}+v^{\mu} \omega_{\mu \nu}^{\prime}\right) \psi^{\nu} \zeta-\frac{\psi^{\nu_{1}} \cdots \psi^{\nu_{4}}}{4 !}\left(v^{\prime} \sigma_{\mu \nu_{1} \cdots \nu_{4}}+v^{\mu} \beta_{\mu \nu_{1} \cdots \nu_{4}}-6 \omega_{\nu_{1} \nu_{2}} \omega_{\nu_{3} \nu_{4}}^{\prime}\right) \\
& =-\left(\iota_{v^{\prime}} \omega+\iota_{v} \omega^{\prime}\right)+\left(-\iota_{v^{\prime}} \sigma-\iota_{v} \beta+\omega \wedge \omega^{\prime}\right)=A \times_{N} A^{\prime}
\end{aligned}
$$

Therefore, the universal bracket formula (1.12) gives (for $\operatorname{deg} \epsilon=\operatorname{deg} \eta=0$ )

$$
\left\{\langle A \mid \epsilon\rangle,\left\langle A^{\prime} \mid \eta\right\rangle\right\}=-\left\langle L_{A} A^{\prime} \mid \epsilon \eta\right\rangle+\left\langle A \times_{N} A^{\prime} \mid \eta d \epsilon\right\rangle .
$$

\footnotetext{
${ }^{7}$ While (3.53) is valid regardless of the form of the 6-dimensional worldvolume metric, in general the canonical variables appearing here will have a more complicated relation to the 3 -form $H$.

${ }^{8}$ See [51], but note that this $N$ is smaller than the one appearing in [51] for $d=6$.
} 
By taking the degree zero $\left.\right|_{\operatorname{deg} 0}$ parts of all superfields, this reduces to the Poisson bracket formula given by Hatsuda et al. [13] for the M5-brane currents

$$
\begin{aligned}
& \left.\langle A \mid \epsilon\rangle\right|_{\operatorname{deg} 0}= \\
& \int_{\Sigma} d^{5} \sigma \epsilon(\sigma)\left(v^{\mu}(X) P_{\mu}+\frac{1}{2} \omega_{\mu \nu}(X) \partial_{\alpha_{1}} X^{\mu} \partial_{\alpha_{2}} X^{\nu} \zeta^{\alpha_{1} \alpha_{2}}+\frac{1}{5 !} \varepsilon^{\alpha_{1} \ldots \alpha_{5}} \sigma_{\mu_{1} \ldots \mu_{5}}(X) \partial_{\alpha_{1}} X^{\mu_{1}} \ldots\right) .
\end{aligned}
$$

The current algebra construction therefore selects the worldvolume 5-form variable

$$
Z_{M} \equiv\left(\begin{array}{c}
P_{\mu} \\
\zeta^{\alpha_{1} \alpha_{2}} \partial_{\alpha_{1}} X^{\mu} \partial_{\alpha_{2}} X^{\nu} \\
\varepsilon^{\alpha_{1} \ldots \alpha_{5}} \sigma_{\mu_{1} \ldots \mu_{5}}(X) \partial_{\alpha_{1}} X^{\mu_{1}} \cdots \partial_{\alpha_{5}} X^{\mu_{5}}
\end{array}\right)
$$

which transforms in the $R_{1}$ representation of $E_{d(d)}$ (for $d=\operatorname{dim} M \leq 6$ ), in a close analogy to expression (1.2) for strings.

The $n<p$-form currents (1.10) correspond directly to the rest of the tensor hierarchy of exceptional generalised geometry/exceptional field theory in M-theory sections; we discuss this in section 4.3 .

Current algebra in the presence of fluxes. We now outline the case where 4- and 7form background fluxes $G$ and $F$ are switched on in (3.39). These lead to very complicated Poisson brackets between $\zeta$ and $P$ (when $G \neq 0$ ) and of $P$ with itself (when $F \neq 0$ or $G \neq 0$; identical to (3.23) when $G=0)$. Those brackets capture the flux contributions to the M5-brane symplectic structure through the Wess-Zumino coupling $\int_{Y} F+G \wedge H$, where $Y$ is a 7-manifold whose boundary is the M5 worldvolume, and $H$ is the field strength of the self-dual gauge field as described above.

The current algebra of e.g. top-form currents corresponding to generalised vectors (3.57) will take the same form as before (again for $\operatorname{deg} \epsilon=\operatorname{deg} \eta=0$ ):

$$
\left\{\langle A \mid \epsilon\rangle,\left\langle A^{\prime} \mid \eta\right\rangle\right\}=-\left\langle L_{A} A^{\prime} \mid \epsilon \eta\right\rangle+\left\langle A \times_{N} A^{\prime} \mid \eta d \epsilon\right\rangle
$$

where $L_{A} A^{\prime}$ will now be the twisted generalised Lie derivative where $F$ and $G$ appear explicitly (see e.g. [52], appendix E.1): indeed the flux contributions in (3.39) yield

$$
\left(A^{\prime}, Q A\right)=\left(A^{\prime}, Q_{0} A\right)+\left(\iota_{v^{\prime}} \iota_{v} G\right) \zeta+\left(\iota_{v^{\prime}} \iota_{v} F-\iota_{v^{\prime}}(G \wedge \omega)+\left(\iota_{v} G\right) \wedge \omega^{\prime}\right)
$$

where $\left(A^{\prime}, Q_{0} A\right)$ is the flux-free contribution to the generalised Lie derivative of formula (3.59).

The ZL reduction will also be affected by the fluxes, similarly to (3.14). For $\zeta$ in particular the ZL condition is

$$
d \boldsymbol{\zeta}=\frac{1}{4 !} G_{\mu_{1} \mu_{2} \cdots \mu_{4}}(\boldsymbol{x}) \boldsymbol{\psi}^{\mu_{1}} \boldsymbol{\psi}^{\mu_{2}} \cdots \boldsymbol{\psi}^{\mu_{4}} .
$$

This can be solved by again employing a Hodge decomposition to separate $\boldsymbol{\zeta}$ into co-exact and closed polyforms on $\Sigma$. The co-exact component is uniquely fixed by (3.67) in terms of 
the target 4-form flux, while the exact and harmonic components remain as independent worldvolume fields. We thus have the same field content as in the case of vanishing fluxes.

We now outline how to work with the simpler brackets (3.47) even when fluxes are present. If we denote $\Theta_{0}=-\psi^{\mu} p_{\mu}$, we can write the "twist" as a canonical transformation of the target QP manifold $\mathcal{M}$ involving local potentials $C_{3}$ and $C_{6}$ for $G$ and $F$ :

$$
\begin{aligned}
\Theta=e^{\Phi}\left(\Theta_{0}\right), \quad \Phi & =C_{\mu_{1} \mu_{2} \mu_{3}}(x) \psi^{\mu_{1}} \psi^{\mu_{3}} \psi^{\mu_{3}} \zeta+C_{\mu_{1} \cdots \mu_{6}}(x) \psi^{\mu_{1}} \cdots \psi^{\mu_{6}} \\
e^{\Phi}(f) & \equiv f+(\Phi, f)+\frac{1}{2}(\Phi,(\Phi, f))+\ldots
\end{aligned}
$$

The transformation $e^{\Phi}$ preserves the $\mathcal{M}$ bracket $(\bullet, \bullet)$. Therefore, for $Q_{0} \equiv\left(\Theta_{0}, \bullet\right)$,

$$
\left\{\left\langle e^{\Phi} f \mid \epsilon\right\rangle,\left\langle e^{\Phi} g \mid \eta\right\rangle\right\}=(-1)^{\epsilon(g+p)}\left\langle e^{\Phi}\left(f, Q_{0} g\right) \mid \epsilon \eta\right\rangle+(-1)^{\epsilon(g+p+1)+g}\left\langle e^{\Phi}(f, g) \mid \epsilon d \eta\right\rangle
$$

from which we find e.g. $\left\{\left\langle e^{\Phi} \chi \mid \epsilon\right\rangle,\left\langle e^{\Phi} \chi \mid \eta\right\rangle\right\}=0$. We infer thereby a field redefinition involving the potentials that expresses all currents in terms of variables with simple (zeroflux) Poisson bracket relations (3.47). When the 4- and 7 -form fluxes $G$ and $F$ are nontrivial, this redefinition will only be valid locally.

The above provides an indirect check that the Poisson brackets of our construction indeed capture the effects of the 4- and 7-form fluxes on the M5 brane symplectic structure: since the zero-flux brackets (3.47) were explicitly checked to match the M5 brane ones (for the degree-zero component fields $X^{\mu}, P_{\mu}$ and $\zeta^{\alpha_{1} \alpha_{2}}$ ), we can imagine introducing flux by a (local) field redefinition involving, say, the 3 -form potential alone:

$$
\begin{aligned}
X^{\mu} \rightarrow X^{\mu}, \quad P_{\mu} & \rightarrow P_{\mu}+\zeta^{\alpha_{1} \alpha_{2}} C_{\mu \nu_{1} \nu_{2}} \partial_{\alpha_{1}} X^{\nu_{1}} \partial_{\alpha_{2}} X^{\nu_{2}}+\mathcal{O}\left(C^{2}\right), \\
\zeta^{\alpha_{1} \alpha_{2}} & \rightarrow \zeta^{\alpha_{1} \alpha_{2}}+C_{\mu_{1} \mu_{2} \mu_{3}} \partial_{\beta_{1}} X^{\mu_{1}} \partial_{\beta_{2}} X^{\mu_{2}} \partial_{\beta_{3}} X^{\mu_{3}} \varepsilon^{\alpha_{1} \alpha_{2} \beta_{1} \beta_{2} \beta_{3}} .
\end{aligned}
$$

This ansatz is the unique one that only involves the 3 -form and preserves the fact $\zeta$ and $P$ are densities of weight 1 . The numerical coefficients and the order $C^{2}$ terms can be determined by demanding that this preserves the zero-flux brackets (3.47) whenever the 3form $C$ is closed. When $C$ is not closed, this transformation does not preserve the Poisson brackets; it introduces a 4 -form flux $G=d C$ and 7-form flux $F=C \wedge d C / 2$. This is precisely the effect of the transformation $e^{\Phi}$ on the target-space QP manifold $\mathcal{M}$ (in the special case where the 6 -form potential is set to zero).

\subsection{D3 brane}

The target QP manifold is:

$$
\mathcal{M}=T^{\star}[4] T[1] M \times \mathbb{R}^{2}[2]
$$

with local homogeneous (in degree) coordinates

$$
\begin{array}{c|c|c|c|c|c|}
\operatorname{coord} & x^{\mu} & \psi^{\mu} & \zeta_{i} & \chi_{\mu} & p_{\mu} \\
\operatorname{deg} & 0 & 1 & 2 & 3 & 4
\end{array}
$$


and nonvanishing Poisson brackets (where $\varepsilon_{j i}=-\varepsilon_{i j}$ and $i, j=1,2$ )

$$
\left(x^{\mu}, p_{\nu}\right)=-\left(p_{\nu}, x^{\mu}\right)=\delta_{\nu}^{\mu}, \quad\left(\psi^{\mu}, \chi_{\nu}\right)=\left(\chi_{\nu}, \psi^{\mu}\right)=\delta_{\nu}^{\mu}, \quad\left(\zeta_{i}, \zeta_{j}\right)=\varepsilon_{i j}
$$

The associated symplectic form therefore has degree $P=4$, whence $p=3$. $P$ is even, so this is quite similar to the previous case. The target space Q-structure $Q$ has the hamiltonian

$$
\Theta=-\psi^{\mu} p_{\mu}-\frac{1}{2} A_{\mu}^{i j} \zeta_{i} \zeta_{j} \psi^{\mu}-\frac{1}{3 !} G_{\mu_{1} \mu_{2} \cdots \mu_{3}}^{i}(x) \psi^{\mu_{1}} \psi^{\mu_{2}} \psi^{\mu_{3}} \zeta_{i}-\frac{1}{5 !} F_{\mu_{1} \mu_{2} \cdots \mu_{5}}(x) \psi^{\mu_{1}} \psi^{\mu_{2}} \cdots \psi^{\mu_{5}}
$$

and $Q$ squares to zero when the background fields satisfy

$$
d A^{i j}+\frac{1}{2} A^{i k} \wedge A^{\ell j} \varepsilon_{k \ell}=0, \quad d G^{i}+G^{j} \wedge A^{k i} \varepsilon_{j k}=0, \quad d F+\frac{1}{2} G^{i} \wedge G^{j} \varepsilon_{i j}=0 .
$$

Therefore the 1-forms $A^{i j}=A^{j i}$ define a flat $\mathrm{SL}(2 ; \mathbb{R})$ connection, the 5 -form $F$ is closed, and the pair $G^{i}$ of 3 -forms are annihilated by the exterior covariant derivative associated to $A^{i j}$. These background fields are related by a redefinition to the type IIB axiodilaton, the Ramond-Ramond 5-form flux, and to the SL(2)-doublet of 3-form fluxes. See [10] for details.

The difference with respect to the generic $p$-brane situation of section 3.2 is the sl(2)-doublet superfield $\boldsymbol{\zeta}_{i}$ which we expand in component fields similarly to what we did for the M5:

$$
\boldsymbol{\zeta}_{i}(\sigma, d \sigma)=\sum_{n=0}^{3} \frac{1}{n !} \zeta_{i \alpha_{1} \cdots \alpha_{n}}(\sigma) d \sigma^{\alpha_{1}} \cdots d \sigma^{\alpha_{n}} \Longrightarrow \operatorname{deg} \zeta_{\alpha_{1} \cdots \alpha_{n}}=2-n .
$$

It is again convenient to work with the dualised component fields

$$
\zeta_{i}^{\alpha_{1} \cdots \alpha_{n}}=\frac{1}{(3-n) !} \varepsilon^{\alpha_{1} \cdots \alpha_{n} \beta_{1} \cdots \beta_{3-n}} \zeta_{i \beta_{1} \cdots \beta_{3-n}} \Longrightarrow \operatorname{deg} \zeta_{i}^{\alpha_{1} \cdots \alpha_{n}}=-1+n
$$

Poisson brackets for vanishing fluxes. In this case $\Theta=-\psi^{\mu} p_{\mu}$ and the ZL reduction reads

$$
d \boldsymbol{x}^{\mu}=\boldsymbol{\psi}^{\mu}, \quad d \boldsymbol{\chi}_{\mu}=-\boldsymbol{p}_{\mu}, \quad d \boldsymbol{\zeta}_{i}=0 .
$$

The independent superfields are again $\boldsymbol{x}^{\mu}$ and $\chi_{\mu}$, but also $\boldsymbol{\zeta}_{i}$, which is constrained to be closed:

$$
d \boldsymbol{\zeta}_{i}=0 \Longleftrightarrow \partial_{\left[\alpha_{1}\right.} \zeta_{\left.|i| \alpha_{2} \cdots \alpha_{n+1}\right]}=0 \Longleftrightarrow \partial_{\alpha} \zeta_{i}^{\alpha \beta_{1} \cdots \beta_{n}}=0 .
$$

Their non-vanishing Poisson brackets are

$$
\begin{aligned}
\left\{x_{\alpha_{1} \alpha_{2} \cdots \alpha_{n}}^{\mu}\left(\sigma_{1}\right), P_{\nu}^{\beta_{1} \beta_{2} \cdots \beta_{m}}\left(\sigma_{2}\right)\right\} & =n !(-1)^{n(p-n)} \delta_{m n} \delta_{\nu}^{\mu} \delta_{\alpha_{1} \alpha_{2} \cdots \alpha_{n}}^{\beta_{1} \beta_{2} \cdots \beta_{m}} \delta^{3}\left(\sigma_{1}-\sigma_{2}\right), \\
\left\{\zeta_{i}^{\alpha_{1} \cdots \alpha_{n}}\left(\sigma_{1}\right), \zeta_{j}^{\beta_{1} \cdots \beta_{m}}\left(\sigma_{2}\right)\right\} & =\varepsilon_{i j} \varepsilon^{\alpha_{1} \cdots \alpha_{n} \gamma \beta_{1} \cdots \beta_{m}} \frac{\partial}{\partial \sigma_{1}^{\gamma}} \delta^{3}\left(\sigma_{1}-\sigma_{2}\right) \delta_{m, 2-n}
\end{aligned}
$$

where the last one in particular is determined from $\left\{\left\langle\zeta_{i} \mid \epsilon\right\rangle,\left\langle\zeta_{j} \mid \eta\right\rangle\right\}=\left\langle\varepsilon_{i j} \mid \epsilon d \eta\right\rangle$. (The other component fields are defined in (3.17) and (3.12) in terms of $\boldsymbol{x}^{\mu}$ and $\boldsymbol{\chi}_{\mu}$.) 
D3-brane phase space and manifest SL(2). The D3 brane has a single U(1) gauge field on its worldvolume. The usual phase space for the bosonic sector of the D3 brane therefore involves the canonical Poisson brackets

$$
\left\{X^{\mu}\left(\sigma_{1}\right), P_{\nu}\left(\sigma_{2}\right)\right\}=\delta_{\nu}^{\mu} \delta^{3}\left(\sigma_{1}-\sigma_{2}\right), \quad\left\{A_{\alpha}\left(\sigma_{1}\right), D^{\beta}\left(\sigma_{2}\right)\right\}=\delta_{\alpha}^{\beta} \delta^{3}\left(\sigma_{1}-\sigma_{2}\right),
$$

where $A_{\alpha}$ are the three spatial components of the 4-potential. The temporal component $A_{0}$ enters as the lagrange multiplier enforcing the Gauss law $\partial_{\alpha} D^{\alpha}=0$. Therefore, upon identifying

$$
\zeta_{1}^{\alpha}=\varepsilon^{\alpha \beta_{1} \beta_{2}} \partial_{\beta_{1}} A_{\beta_{2}}, \quad \zeta_{2}^{\alpha}=D^{\alpha},
$$

we see from (3.79) that the D3 brane phase space is identical to the degree-zero non-degenerate subspace produced by the current algebra construction, where the constraint (3.78) has been enforced. (The harmonic components of $\boldsymbol{\zeta}_{i}$ span a degenerate subspace with respect to the Poisson bracket, similar to what we saw for the M5 brane.)

Since this non-degenerate subspace for the fields $\boldsymbol{\zeta}_{i}$ consists of exact fields, we could also solve (3.78) to arrive at an SL(2)-doublet of potentials. That formulation of the D3brane phase space was recently arrived at by Mezincescu and Townsend ([53], section 3.1.1) in the context of the tensionless limit, for which the dynamics on any IIB background and not just the phase space - enjoys an SL(2)-invariance as well.

Current algebra (for vanishing fluxes). Top-form currents (1.10) of degree zero arise from $A \in C^{\infty}(\mathcal{M})$ of degree $p=3$ :

$$
-A=v^{\mu}(x) \chi_{\mu}+\lambda_{\mu}^{i}(x) \psi^{\mu} \zeta_{i}+\frac{1}{3 !} \rho_{\mu \nu \rho}(x) \psi^{\mu} \psi^{\nu} \psi^{\rho} .
$$

This corresponds to a section of the bundle $E=\left(T \oplus T^{\star} \oplus T^{\star} \oplus \Lambda^{3} T^{\star}\right) M$ of generalised vectors for $E_{5} \cong \operatorname{Spin}(5,5)$ exceptional generalised geometry (for $\operatorname{dim} M=4$ ) or $E_{d}=$ $\mathrm{SL}(5), \mathrm{SL}(3) \times \mathrm{SL}(2), \ldots$ (for $d=3,2, \ldots)$, in a type IIB section $[49,50]$. As for the M5 brane, other values of $d$ are allowed, but $E_{d+1}$-covariance will break. The expressions $\left(A^{\prime},(Q A)\right)$ and $\left(A, A^{\prime}\right)$ that determine the current algebra were explicitly calculated in [10] and again correspond to the type IIB generalised Lie derivative $L_{A} A^{\prime}$ and the symmetric map $\times_{N}: E \otimes E \rightarrow N \equiv \Lambda^{2} T^{\star} M \oplus \mathbb{R}^{2}$. Therefore, the universal bracket formula (1.12) gives (for $\operatorname{deg} \epsilon=\operatorname{deg} \eta=0$ )

$$
\left\{\langle A \mid \epsilon\rangle,\left\langle A^{\prime} \mid \eta\right\rangle\right\}=-\left\langle L_{A} A^{\prime} \mid \epsilon \eta\right\rangle+\left\langle A \times_{N} A^{\prime} \mid \eta d \epsilon\right\rangle .
$$

By restricting to the zero degree $\left.\right|_{\operatorname{deg} 0}$ parts of all currents, we deduce that the algebra of D3-brane currents built from the worldvolume 3 -form variable

$$
Z_{M} \equiv\left(\begin{array}{c}
P_{\mu} \\
\zeta_{i}^{\alpha} \partial_{\alpha} X^{\mu} \\
\varepsilon^{\alpha_{1} \alpha_{2} \alpha_{3}} \partial_{\alpha_{1}} X^{\mu_{1}} \partial_{\alpha_{2}} X^{\mu_{2}} \partial_{\alpha_{3}} X^{\mu_{3}}
\end{array}\right)
$$

has a geometric expression in terms of exceptional generalised geometry, as was found by Hatsuda et al. [14]. 
The $n$-form currents for $0 \leq n<p$ correspond to sections of bundles of the type IIB tensor hierarchy; see section 4.3 .

\section{Applications}

\subsection{Brane dynamics}

The construction of section 2 produces a Poisson algebra, which, under favourable conditions, contains (in its degree-zero subspace) the phase space appearing in the usual hamiltonian formulation of brane physics. This defines the brane kinematics. Could we say anything about brane dynamics?

Surprisingly, the answer is yes, at least when the brane dynamics is reparameterisationinvariant, i.e. invariant under worldvolume diffeomorphisms. This is the case for the branes of relevance in string/M-theory, including the string. In the hamiltonian formulation, reparameterisation invariance is realised thus: the hamiltonian (density) $H$ is a collection of constraint functions $\mathcal{H}, \mathcal{H}_{\alpha}$ :

$$
H=e \mathcal{H}+u^{\alpha} \mathcal{H}_{\alpha},
$$

where $\mathcal{H}$ and $\mathcal{H}_{\alpha}$ respectively generate temporal and spatial diffeomorphisms via the Poisson bracket. The corresponding lagrange multipliers $e$ and $u^{\alpha}$ are a scalar density and a spatial vector field. ( $\alpha=1, \ldots, p$ is again a worldspace index.) When the constraint algebra is closed - first-class - under Poisson brackets, worldvolume diffeomorphisms are realised as gauge symmetries. This algebra is rather similar to that of the hamiltonian and momentum constraints in the $\mathrm{ADM}$ formulation of general relativity; in particular, the spatial constraints $\left(\mathcal{H}_{\alpha}\right)$ form a closed subalgebra corresponding to infinitesimal spatial diffeomorphisms. (There might also be further constraints when worldvolume gauge fields are present, depending on our choice of phase space variables; they do not arise here.)

If we fix a phase space along with $\mathcal{H}$ and $\mathcal{H}_{\alpha}$, we have completely specified the reparameterisation-invariant brane dynamics. The brane phase space construction actually provides a model-independent definition for the spatial constraints $\mathcal{H}_{\alpha}$, at least for the standard choice of real source Q-manifold

$$
\mathcal{N}=T[1] \Sigma
$$

where $\Sigma$ is a spatial section of the worldvolume. The only remaining choice will be the hamiltonian constraint $\mathcal{H}$.

\subsubsection{The spatial diffeomorphism constraints}

We saw above that the de Rham differential $d$ is interpreted as a vector field on $\mathcal{N}=T[1] \Sigma$. In fact the same is true for the interior product $\iota_{v}$ and Lie derivative $\mathcal{L}_{v}$ associated to any vector field $v^{\alpha}(\sigma)$ on $\Sigma$. We therefore have a natural candidate for the corresponding spatial diffeomorphism generator: the hamiltonian associated to the vector field $\mathcal{L}_{v}$ (if it exists). We will see now that $\mathcal{L}_{v}$ - appropriately understood - is a hamiltonian vector field for both the original $[\bullet, \bullet]$ and ZL-reduced bracket $\{\bullet, \bullet\}$. 
Consider first the general setting of zero locus reduction of a Poisson algebra $(\mathcal{P},[\bullet, \bullet])$ with respect to a Q-structure $Z$ as described in Proposition 2. Then

Lemma 1. If

$$
X \equiv(-1)^{X}\left[Z\left(\Phi_{X}\right), \bullet\right]
$$

for $\Phi_{X} \in \mathcal{P}$ of definite degree, then $X$ descends to a hamiltonian derivation $\tilde{X}$ on the zero locus:

$$
\tilde{X}(\tilde{F}) \equiv \widetilde{X(F)}=\left\{\tilde{\Phi}_{X}, \tilde{F}\right\},
$$

where the tildes denote the zero locus quotient of Proposition 2.

We set

$$
X=\overline{\mathcal{L}}_{v}
$$

where the bar again means we lift the Lie derivative $\mathcal{L}_{v}$ to the space of maps $\operatorname{Maps}(T[1] \Sigma \rightarrow$ $\mathcal{M}$ ). Since $Z=\bar{d}-\bar{Q}$, we can use Cartan's magic formula to find

$$
X=\overline{\left[\iota_{v}, d\right]}=-\left[\bar{\iota}_{v}, \bar{d}\right]=-\left(\bar{\iota}_{v} Z+Z \bar{\iota}_{v}\right)
$$

where we exploited the fact $\bar{Q}$ (the lift of the target-space $\mathcal{M}$ vector $Q$ to Maps) (anti)commutes with all lifts of source-space $T[1] \Sigma$ vector fields. (See the appendix for the minus sign.) If $-\bar{\iota}_{v}$ is hamiltonian for $[\bullet, \bullet]$ with hamiltonian function $\Phi_{v}$, it follows that $\overline{\mathcal{L}}_{v}=\left[Z \Phi_{v}, \bullet\right]$, so the lemma applies.

The vector field $\bar{\iota}_{v}$ is indeed hamiltonian because of three familiar facts:

1. the measure $\int_{T[1] \Sigma}$ is invariant: $\int_{T[1] \Sigma} \iota_{v} \epsilon=0$ (because the integral picks out the top-form component);

2. the Poisson structures $(\bullet, \bullet)$ and $[\bullet, \bullet]$ on the target space $\mathcal{M}$ and mapping space $\operatorname{Maps}(T[1] \Sigma \rightarrow \mathcal{M})$ are both nondegenerate, and the symplectic forms are exact;

3. any vector field that annihilates the symplectic potential is hamiltonian.

In our conventions, if $\Omega=\delta \Xi$ is the (odd) symplectic form associated to $[\bullet, \bullet]$ and $\mathcal{L}_{X} \Xi=0$, its associated hamiltonian is the contraction $\iota_{X} \Xi$. For the target-space symplectic form $\omega$, we select the canonical potential $\xi=P^{-1} \iota_{E} \omega$ involving the degree-counting "Euler" vector field $E$, which induces via transgression the following 1-form $\Xi$ on Maps:

$$
\Xi=\int_{T[1] \Sigma} \frac{1}{P}\left(\operatorname{deg} z^{a}\right) \boldsymbol{z}^{a} \omega_{a b}(\boldsymbol{z}) \delta \boldsymbol{z}^{b}
$$

Since deg $\iota_{\bar{\iota}_{v}}=-2$, we find

$$
\Phi_{v} \equiv \iota_{-\bar{\iota}_{v}} \Xi=-\int_{T[1] \Sigma} \frac{1}{P}\left(\operatorname{deg} z^{a}\right) \boldsymbol{z}^{a} \omega_{a b}(\boldsymbol{z}) \iota_{v} \boldsymbol{z}^{b} .
$$

We refer to ([10], appendix A, B) and to the appendix here for the conventions that lead to these formulas. It is clear from the above explicit expression that $\mathcal{L}_{\bar{\iota}_{v}} \Xi=0$. 
By construction, the image $\tilde{\Phi}_{v}$ of $\Phi_{v}$ under the zero-locus quotient is the hamiltonian that generates an infinitesimal spatial diffeomorphism (associated to the vector field $v$ on $\Sigma)$. In general, the ZL reduction will introduce a dependence on the background fluxes inside $\tilde{\Phi}_{v}$ whenever the fluxes correspond to nontrivial classes (that contribute to the brane symplectic form). This dependence can be removed by a local field redefinition to match the more familiar fluxless generators, as in the discussion around (3.70).

Check: the M5 brane. The canonical potential ${ }^{9}$ associated to the target-space $\mathcal{M}=$ $T^{\star}[6] T[1] M \times \mathbb{R}[3]$ symplectic form $\omega$ whose Poisson structure is defined by (3.38) is

$$
\xi=p_{\mu} d x^{\mu}-\frac{1}{6}\left(5 \chi_{\mu} d \psi^{\mu}+\psi^{\mu} d \chi_{\mu}\right)-\frac{1}{2} \zeta d \zeta
$$

Therefore

$$
\Phi_{v}=-\int_{T[1] \Sigma} \boldsymbol{p}_{\mu} \iota_{v} \boldsymbol{x}^{\mu}-\frac{5}{6} \boldsymbol{\chi}_{\mu} \iota_{v} \boldsymbol{\psi}^{\mu}-\frac{1}{6} \boldsymbol{\psi}_{\mu} \iota_{v} \boldsymbol{\chi}_{\mu}-\frac{1}{2} \boldsymbol{\zeta} \iota_{v} \boldsymbol{\zeta}
$$

For the purposes of comparison we switch off the possible 4-form and 7-form fluxes in (3.39). Therefore the ZL reduction is given by (3.45). Using that to eliminate $\boldsymbol{p}_{\mu}$ and $\boldsymbol{\psi}^{\mu}$, we calculate (using integration by parts, where we dropped the tilde notation on the righthand side)

$$
\tilde{\Phi}_{v}=\int_{T[1] \Sigma} \boldsymbol{\chi}_{\mu}\left(\iota_{v} d+d \iota_{v}\right) \boldsymbol{x}^{\mu}-\frac{1}{2} \boldsymbol{\zeta} \iota_{v} \boldsymbol{\zeta}=\int_{T[1] \Sigma} \boldsymbol{\chi}_{\mu} \mathcal{L}_{v} \boldsymbol{x}^{\mu}-\frac{1}{2} \boldsymbol{\zeta} \iota_{v} \boldsymbol{\zeta}
$$

The degree-zero part $\left.\tilde{\Phi}_{v}\right|_{\operatorname{deg} 0}$ is (NB (3.17) and (3.42))

$$
\left.\tilde{\Phi}_{v}\right|_{\operatorname{deg} 0}=\int_{\Sigma} d^{5} \sigma-v^{\alpha}\left(P_{\mu} \partial_{\alpha} X^{\mu}+\frac{1}{2} \zeta^{\beta_{1} \beta_{2}} \zeta_{\alpha \beta_{1} \beta_{2}}\right) .
$$

We read off the usual M5-brane spatial diffeomorphism generator:

$$
\mathcal{H}_{\alpha}=P_{\mu} \partial_{\alpha} X^{\mu}+\frac{1}{2} \zeta^{\beta_{1} \beta_{2}} \zeta_{\alpha \beta_{1} \beta_{2}}
$$

By comparing with the explicit form of the Poisson brackets (3.47), we see the bracket $\left\{\left.\tilde{\Phi}_{v}\right|_{\operatorname{deg} 0}, \bullet\right\}$ indeed generates the infinitesimal diffeomorphism parameterised by the vector field $v^{\alpha}$ on the worldspace $\Sigma$. (For this check it is necessary to use the fact that $\zeta$ is closed: $\left.\partial_{\alpha} \zeta^{\alpha \beta}=0.\right)$

\subsubsection{The hamiltonian constraint}

The hamiltonian constraint $\mathcal{H}$ actually generates diffeomorphisms in worldvolume time up to trivial gauge transformations, meaning up to gauge transformations that vanish when the equations of motion are used. It is probably for that reason that the method that worked for $\mathcal{H}_{\alpha}$ does not seem to apply to produce a formula for $\mathcal{H}$.

\footnotetext{
${ }^{9}$ Note in [10] we used a different convention for the canonical potential: the one there satisfies $-d \vartheta=\omega$, whereas here $d \xi=\omega$.
} 
Nevertheless, the hamiltonian constraint does apparently have a universal expression. ${ }^{10}$ If $\mathcal{M}^{M N}$ is the inverse generalised metric, then

$$
\mathcal{H}=\mathcal{M}^{M N} Z_{M} Z_{N}
$$

where $Z_{M}$ is the local basis of $p$-form currents (that correspond to a local basis of sections of the generalised tangent bundle). For the string these are the familiar expressions $Z_{M}=$ $\left(P_{\mu}, \partial_{\sigma} X^{\mu}\right)$ given in the Introduction, while for the M5 and D3 branes these were written down in (3.64) and (3.84).

The notion of a generalised metric has not yet been formulated in a way that usefully interacts with the QP structure, except for the familiar case of degree $P=2$ (i.e. Courant) algebroids: there, a generalised metric is identified with a symplectomorphism $\mathcal{M}$ that fixes the body of the QP manifold and squares to +1 [56]. Even in this case it is not yet clear how to reverse-engineer a prescription that will produce (4.13).

There is however a considerable simplification relevant to our construction. Since the fluxes are present in the Q-structure and therefore inside the twisted generalised Lie derivative, we are in the "untwisted" picture (in the terminology of $[49,52]$ ). The potentials cannot also appear inside the generalised metric, because we would be counting them twice. Therefore, when we account for the fluxes by inserting them in the Q-structure (as in formula (3.39) for the M5 brane), we must use the fluxless expression for the generalised metric, which is rather simple. For the M5 brane for example (where combinatorial factors were ignored),

$$
\mathcal{M}^{M N}=\left(\begin{array}{lll}
g^{\mu \nu} & & \\
& g_{\mu[\nu} g_{\rho] \sigma} & \\
& & g_{\mu_{1}\left[\nu_{1}\right.} g_{\left|\mu_{2}\right| \nu_{2}} \cdots g_{\left.\nu_{5}\right] \mu_{5}}
\end{array}\right),
$$

where $g_{\mu \nu}$ is the spacetime metric.

\subsection{Dirac structures and the 't Hooft anomaly}

If we have a sigma model with target $M$, isometries of $M$ usually correspond to global symmetries of the sigma model. We consider the problem of gauging those symmetries to obtain a sigma model whose target is the space of orbits. This procedure is not always consistent; when it fails, there is a 't Hooft anomaly. (More generally, we say such an anomaly occurs whenever there is an obstruction to gauging some global symmetry. See e.g. the review section in [57].) It is well-known that such anomalies can occur even for classical theories e.g. in the presence of Wess-Zumino terms describing electric flux couplings to the brane [58], and for Wess-Zumino terms related to cocycles for the supertranslation group [59].

A nice feature of the universal bracket formula (1.12) is that it translates the obstruction on the sigma model to a geometric condition on the target QP manifold, which can be understood in generalised or exceptional generalised geometry (for those branes relevant in string/M-theory).

\footnotetext{
${ }^{10}$ See $[13,54]$ for the M2 and M5, and [40] for a more exotic example. For the M2, this was originally pointed out by Berman and Perry [55].
} 
We illustrate using the M5 brane. We will need some previously-derived expressions, starting from the current arising from a degree $p=5$ function $A \in C^{\infty}(\mathcal{M})$ :

$$
\begin{aligned}
A & =-v^{\mu}(x) \chi_{\mu}+\omega \zeta+\sigma \\
\left.\langle A \mid \epsilon\rangle\right|_{\operatorname{deg} 0} & = \\
\int_{\Sigma} d^{5} \sigma \epsilon(\sigma) & \left(v^{\mu}(X) P_{\mu}+\frac{1}{2} \omega_{\mu \nu}(X) \partial_{\alpha_{1}} X^{\mu} \partial_{\alpha_{2}} X^{\nu} \zeta^{\alpha_{1} \alpha_{2}}+\frac{1}{5 !} \varepsilon^{\alpha_{1} \ldots \alpha_{5}} \sigma_{\mu_{1} \ldots \mu_{5}}(X) \partial_{\alpha_{1}} X^{\mu_{1}} \ldots\right)
\end{aligned}
$$

corresponding to the generalised vector $A=v+\omega+\sigma \in \Gamma[E]$, and its colleague $\left.\left\langle A^{\prime} \mid \eta\right\rangle\right|_{\operatorname{deg} 0}$. If we were to gauge both currents, the necessary and sufficient condition for (classical) consistency under time evolution is that their Poisson bracket is zero modulo the constraints. We will treat the case where the M5 brane is coupled to 4 -form and 7-form fluxes $G$ and $F$; this corresponds to the Q-structure given in (3.39), which leads to the expressions

$$
\begin{aligned}
\left(A^{\prime}, Q A\right)=\mathcal{L}_{v} v^{\prime}+ & \left(\mathcal{L}_{v} \omega^{\prime}-\iota_{v^{\prime}} d \omega\right) \zeta & +\left(\mathcal{L}_{v} \sigma^{\prime}-\iota_{v^{\prime}} d \sigma-\omega^{\prime} \wedge d \omega\right) \\
& +\left(\iota_{v^{\prime}} \iota_{v} G\right) \zeta & +\iota_{v^{\prime}} \iota_{v} F-\iota_{v^{\prime}}(G \wedge \omega)+\left(\iota_{v} G\right) \wedge \omega^{\prime},
\end{aligned}
$$

which is the twisted generalised Lie derivative $L_{A} A^{\prime}$, and

$$
\left(A, A^{\prime}\right)=-\left(\iota_{v^{\prime}} \omega+\iota_{v} \omega^{\prime}\right) \zeta+\left(-\iota_{v^{\prime}} \sigma-\iota_{v} \sigma^{\prime}+\omega \wedge \omega^{\prime}\right),
$$

which is the symmetric map $\times_{N}: E \otimes E \rightarrow N$ familiar from exceptional generalised geometry. The universal bracket formula then specifies the Poisson bracket of currents:

$$
\left\{\langle A \mid \epsilon\rangle,\left\langle A^{\prime} \mid \eta\right\rangle\right\}=-\left\langle\left(A^{\prime}, Q A\right) \mid \epsilon \eta\right\rangle+\left\langle\left(A, A^{\prime}\right) \mid \eta d \epsilon\right\rangle .
$$

Consider now a collection of vector fields $v_{i} \in \Gamma[T M]$ that forms a Lie algebra,

$$
\left[v_{i}, v_{j}\right] \equiv \mathcal{L}_{v_{i}} v_{j}=f_{i j}{ }^{k} v_{k}
$$

with structure constants $f_{i j}{ }^{k}$. These correspond to the degree 5 functions $v_{i} \equiv v_{i}^{\mu} \chi_{\mu}$ on the target QP manifold $\mathcal{M}=T^{\star}[6] T[1] M \times \mathbb{R}[3]$. The associated currents (4.15) satisfy

$$
\left\{\left\langle v_{i} \mid \epsilon\right\rangle,\left\langle v_{j} \mid \eta\right\rangle\right\}=-f_{i j}{ }^{k}\left\langle v_{k} \mid \epsilon \eta\right\rangle+\left\langle\left(\iota_{v_{i}} \iota_{v_{j}} G\right) \zeta+\iota_{v_{i}} \iota_{v_{j}} F \mid \epsilon \eta\right\rangle .
$$

Unless the fluxes are such that $\iota_{v_{i}} \iota_{v_{j}} G=\iota_{v_{i}} \iota_{v_{j}} F=0$, there is an obstruction to gauging these currents. This is true independently of whether the vectors $\left\{v_{i}\right\}$ are Killing. (If they are not, we will find another obstruction when we calculate the Poisson bracket involving the hamiltonian constraint $\mathcal{H}$ of (4.13) and (4.14).)

One can attempt to modify the currents $\left\langle v_{i}\right|$ by introducing 2-form and 5-form components $\omega_{i}, \sigma_{i}$ as in expression (4.15): the infinitesimal action on the brane embedding $X^{\mu}(\sigma)$ will be the same, but the modified currents will act differently on the momenta $P_{\mu}(\sigma)$ and the M5 brane gauge field variable $\zeta^{\alpha \beta}(\sigma)$, ideally in a way that removes the obstruction. At this point we are investigating the action of a generalised vector on the M5 phase space, so 
it is more convenient to adopt a generalised geometry language to succinctly characterise when the obstruction vanishes:

Proposition 3. Let $L$ be a Dirac structure for $E=T \oplus \Lambda^{2} T^{\star} \oplus \Lambda^{5} T^{\star}$ generalised geometry: a subbundle of the generalised tangent bundle $E$, that is closed with respect to the (twisted) generalised Lie derivative $L_{A} A^{\prime}$ (4.16), and isotropic with respect to the bilinear form $\times_{N}$ (4.17). Any local basis of sections of L gives rise to anomaly-free M5-brane currents of the form (4.15).

This particular structure is defined by Tennyson and Waldram [60] following an analogous definition for $E_{7(7)}$ generalised geometry [61]; the Neumann subbundles of the D-brane structures of Blair [62] are also examples.

The most general statement - valid for any $p$-brane — is best phrased in the QP language. For any $\mathrm{QP}$ target $(\mathcal{M}, Q, \omega)$ with degree $P$ symplectic form $\omega$, the bracket

$$
f, g \rightarrow(f, Q g)
$$

that appears in the bracket formula (1.12), relates functions of different degrees unless

$$
\operatorname{deg} f=\operatorname{deg} g=P-1 .
$$

Therefore this must be the subspace where we can accommodate a closed algebra of Killing vectors as in (4.19), independently of the details of $\mathcal{M}$. For the typical source manifold $\mathcal{N}=T[1] \Sigma$ relevant for $p$-branes we have $P-1=\operatorname{dim} \Sigma=p$. This motivates the following general definition:

Definition 1. If $(\mathcal{M}, \omega, Q)$ is a $Q P$ manifold with $\operatorname{deg} \omega=P$, then a Dirac structure $\mathcal{L}$ of $(\mathcal{M}, \omega, Q)$ is a maximal subspace of $C_{P-1}^{\infty}(\mathcal{M})$ (functions of degree $P-1$ ) that is involutive:

$$
f, g \in \mathcal{L} \Longrightarrow(f, Q g) \in \mathcal{L}
$$

and isotropic:

$$
f, g \in \mathcal{L} \Longrightarrow(f, g)=0 .
$$

(The second condition plus the Jacobi identity implies also $(g, Q f) \in \mathcal{L}$.)

Every Dirac structure gives rise to a maximal collection of anomaly-free currents via (1.10).

For $P=2, \mathcal{M}=T^{\star}[2] T[1] M$, we recover the original notion of Dirac structure $[3,4]$ in generalised geometry, that was related to anomaly-free string currents by Alekseev and Strobl [2]. For the cotangent case $\mathcal{M}=T^{\star}[P] \mathcal{M}^{\prime}$, this agrees with the definition of Ikeda and Koizumi ([8], Theorem 7.1) given in the same context. Definition 1 is related to Ševera's $\Lambda$-structures [27], which are lagrangian submanifolds where $Q$ descends ("dg-submanifolds"): if a $\Lambda$-structure defined by a lagrangian submanifold $\mathcal{Y}$ is such that $\mathcal{Y}_{0}=\mathcal{M}_{0}$ - i.e. $\mathcal{M}$ and the submanifold $\mathcal{Y}$ are identical in degree zero - then the $\Lambda$ structure provides a Dirac structure in the above sense. Similar ideas have also appeared in the context of "higher" versions of Hitchin-Gualtieri generalised geometry: in [63], Bursztyn, Alba and Rubio introduce higher Dirac structures for the algebroid $T M \oplus \Lambda^{k} T^{\star} M$. 
These higher Dirac structures would correspond precisely to the ones of Definition 1 for the QP-manifold of section 3.2 (for $k=P-1$ ) if we dropped the maximality condition from Definition $1 .^{11}$

\subsubsection{SUSY backgrounds and exceptional (generalised) complex structures}

Dirac structures for the particular case of M5 branes - i.e. the generalised geometry of the

$$
E=T \oplus \Lambda^{2} T^{\star} \oplus \Lambda^{5} T^{\star}
$$

bundle - arise naturally in $N=1$ supersymmetric M-theory flux compactifications to $D=5$ Minkowski space. These structures are the output of a general programme of understanding the Killing spinor conditions as the existence of tensors on $E$ that are stabilised by a group $G$; in other words, in terms of $G$-structures. (See [52, 60, 64, 65] for 5-dimensional Minkowski compactifications.) Most relevant for us are the exceptional complex structures of [60], which encode part of the supersymmetry conditions.

We reproduce the relevant definitions from [60]:

Definition 2. An integrable $\mathbb{R}^{+} \times \mathrm{U}^{*}(6)$ or exceptional complex structure is defined by subbundles $L_{1}, L_{0}$ of the complexified generalised tangent bundle $E_{\mathbb{C}}$ that satisfy

i) $\operatorname{dim}_{\mathbb{C}} L_{1}=6$,

ii) $L_{1} \times_{N} L_{1}=0$,

iii) $L_{1} \cap \bar{L}_{1}=\{0\}$ and $L_{1} \cap L_{0}=\{0\}$,

iv) (omitted here)

along with the integrability condition: $L_{V} U$ is a section of $L_{1}$ if $V, U$ are sections of $L_{1}$.

These imply in particular that

$$
E_{\mathbb{C}}=L_{1} \oplus \bar{L}_{1} \oplus L_{0}
$$

is a splitting of the complexified generalised tangent bundle $E$ into a direct sum of Dirac structures $-L_{1}$ and its complex conjugate $\bar{L}_{1}-$ plus $L_{0}$. This is precisely analogous to the result of [2] for strings and generalised complex structures [66], except in the string case there is no complementary bundle $L_{0}$.

The upshot is that the supersymmetry conditions for the above compactification manifold endow the M5-brane worldvolume with a pair of anomaly-free current subalgebras (that do not mutually commute). We expect a similar relation between supersymmetry and anomaly-free currents to be valid fairly generally, because it is also true that supersymmetric compactifications to e.g. $D=4$ Minkowski space give rise to Dirac structures for $E_{7(7)}$-generalised geometry for both M-theory and type IIB [61].

\footnotetext{
${ }^{11}$ To elaborate: the Bursztyn-Alba-Rubio higher Dirac structures involve involutive, isotropic subbundles which are not lagrangian but instead weakly lagrangian. It is not entirely clear whether weakly lagrangian subbundles are automatically maximal (not included inside a bigger such subbundle).
} 


\begin{tabular}{|c|ccccc|}
\hline$E_{d(d)}$-module & $R_{1}$ & $R_{2}$ & $\ldots$ & $R_{P-1}$ & $R_{P}$ \\
functions on $\mathcal{M}$ of degree & $P-1$ & $P-2$ & $\ldots$ & 1 & 0 \\
$p$-brane currents by differential form rank & $p$ & $p-1$ & $\ldots$ & 1 & 0 \\
\hline
\end{tabular}

Table 2. Tensor hierarchy modules, their corresponding functions on the QP-manifold $\mathcal{M}$, and the associated brane currents (for real source Q-manifold $\mathcal{N}=T[1] \Sigma) . P=p+1$ is the degree of the symplectic structure $\omega$ on $\mathcal{M} ; p=\operatorname{dim} \Sigma$. For certain $n, p, d$, the $R_{p+1-n}$ module is strictly larger than the $n$-form current multiplet, as described in text.

\section{3 $L_{\infty}$-algebras, brane currents, and the tensor hierarchy}

The brane current hierarchy. Any QP manifold $(\mathcal{M}, \omega, Q)$ comes with an infinitedimensional $L_{\infty}$-algebra ${ }^{12}$ that lives on the vector space of functions on $\mathcal{M}$ of degrees up to $\operatorname{deg} \omega=P$ ([28], Theorem 4.4). For the QP manifolds of table 1, this $L_{\infty}$-algebra was later identified as the tensor hierarchy of exceptional generalised geometry/exceptional field theory ${ }^{13}[10]$. The correspondence is given in table 2 .

We saw earlier that the brane phase space construction gives a distinguished class of currents (1.10). The ones that do not vanish when we restrict to deg 0 components of all superfields are precisely those that arise from functions $f \in C^{\infty}(\mathcal{M})$ of degree $<P$. Therefore, the tensor hierarchy gives rise to a brane current hierarchy (table 2). We see that going higher in the tensor hierarchy means going lower in the brane current hierarchy, when the brane currents are ordered by differential-form rank.

"Missing modules". Some of the $E_{d(d)}$-modules of table 2 are only captured partially by functions on $\mathcal{M}$, depending on the rank of the duality group $E_{d(d)}$ and the type of construction (M-theory versus type IIB). The systematic patterns of these "missing modules" are listed in [10]. (More exposition and references on the tensor hierarchy are also given there.)

The worldvolume picture of the tensor hierarchy provides an intuitive explanation for the missing modules. We illustrate with the M2 brane, which is the case $P=3$ of the construction of section 3.2. The top-form currents in this case are the 2-form currents (3.26); if $d \equiv \operatorname{dim} M<5$ these fill out the $R_{1}$ module of the $E_{d(d)}$ tensor hierarchy (i.e. the module of generalised vectors). The next module in the tensor hierarchy is $R_{2}$. On the M2 brane the 1-form currents $\left\langle\lambda_{\mu}(x) \psi^{\mu} \mid \epsilon\right\rangle$ correspond to spacetime 1-forms $\lambda_{\mu}$ :

$$
\int d^{2} \sigma \lambda_{\mu}(X(\sigma)) \varepsilon^{\alpha \beta} \partial_{\alpha} X^{\mu} \epsilon_{\beta}(\sigma)
$$

The 1-form currents on the M2 brane take this form regardless of the dimension of spacetime. Therefore, after a certain large value of $d$ - namely 4 - the $R_{2}$ module of $E_{d(d)}$ will always be larger than the $\mathrm{GL}(d)$ module of 1-forms. For $d=4$ the exceptional group

\footnotetext{
${ }^{12}$ We reviewed $L_{\infty}$-algebras recently in [67]. The degree convention of ([10], Proposition 3) (where the $L_{\infty}$-algebra associated to the QP manifold is given) matches the convention of ([67], section 2).

${ }^{13}$ See [68-70] for non-QP derivations of this $L_{\infty}$-algebra; Cederwall and Palmkvist [69] and Cagnacci, Codina, and Marques [70] in particular both deal with the tensor hierarchy in the ExFT context — including extended coordinates - which is strictly bigger than the tensor hierarchy obtained in the QP construction (which has no extended coordinates).
} 
is $E_{4(4)}=\mathrm{SL}(5)$ and the $R_{2}$ module corresponds to the $\overline{\mathbf{5}}$ representation, which is larger than the module of 1 -forms of the 4-dimensional spacetime.

Therefore the explanation for the patterns of missing modules on the QP-manifold side observed in [10] is that the corresponding branes do not have enough degrees of freedom to accommodate full $E_{d(d)}$ current multiplets. This is physically reasonable since U-duality sends e.g. M2 branes to M5 branes and permutes strings and D-branes; unless we introduce extended coordinates to accommodate brane winding modes, we cannot expect duality rotations to be (fully) realised on the worldvolume, except possibly for low rank duality groups as we just saw for the M2 brane.

$\boldsymbol{L}_{\infty}$-algebras on the brane? The brackets of the $L_{\infty}$-algebra associated to the QP manifold $(\mathcal{M}, \omega, Q)$ take the schematic form

$$
\left[f_{1}, f_{2}, \ldots f_{n}\right] \sim\left(\cdots\left(\left(Q f_{1}, f_{2}\right), f_{3}\right) \cdots f_{n}\right), \quad f_{1}, f_{2} \ldots, f_{n} \in C^{\infty}(\mathcal{M})
$$

which is to be (anti)symmetrised in $1,2, \ldots n$ (see [10], Proposition 3 ). The natural question is: given the correspondence between elements of this $L_{\infty}$-algebra and brane currents (1.10)

$$
f_{i} \rightarrow\left\langle f_{i}\right|
$$

(how) is the $L_{\infty}$-algebraic structure realised on the brane phase space?

We do not yet have an answer, but we do point out that the explicit form of (4.27) suggests strongly that the target-space $L_{\infty}$-algebra brackets can be written down using nested Poisson brackets of the corresponding currents, via the universal bracket formula (1.12).

The relation must necessarily be somewhat indirect, because the current algebra of phase space currents with the Poisson brackets (1.12) is a Lie algebra, while the $L_{\infty^{-}}$ algebra of the tensor hierarchy produced by the QP-structure will be a Lie $P$-algebra, so it will in general have non-vanishing brackets of up to $P+1$ arguments. A natural conjecture is that the relation is through the transgression (of Lie P-algebras to Lie algebras) of Sati and Schreiber [71]: roughly speaking, they produce a Lie algebra from a Lie $P$-algebra by considering the space of embeddings of a $p$-brane worldvolume onto a geometry. This is of course strongly reminiscent of our brane phase space construction. A technical difference is that our $n$-form currents (1.10) are integrated against test $(p-n)$-forms $\epsilon$ to produce an integral over the whole worldspace $\Sigma$, whereas those of [71] are not. It is in the latter formulation that Poisson brackets in field theory and $L_{\infty}$-algebras were originally connected (by Barnich, Fulp, Lada, and Stasheff [72]; see also [73]). Their higher Poisson brackets appear to be connected to the freedom of shifting local functionals by total divergence terms (see also $[74,75]$ ), which must be encoded rather differently in our approach due to the smearing against test forms $\epsilon$.

\section{Acknowledgments}

This paper originated from discussions with David Tennyson and Daniel Waldram, and with Chris Blair, David Osten, and Dan Thompson. I am grateful for continued collaboration 
with Chris Blair and Dan Thompson on a related project that has informed this one, and for their numerous suggestions for the manuscript. I would also like to thank David Tennyson for many clarifications on [60] and for sending me an advance copy of that manuscript, and David Osten for correspondence that helped me understand his recent paper [23]. Finally I would also like to thank Paul K. Townsend for reading the manuscript, for encouragement, and for bringing certain references to my attention.

I would furthermore like to thank David Berman and Noriaki Ikeda for their helpful correspondence following the appearance of the original manuscript on the arXiv.

I am supported in part by the "FWO-Vlaanderen" through the project G006119N and by the Vrije Universiteit Brussel through the Strategic Research Program "High-Energy Physics".

\section{A Sign manifesto, conventions, the AKSZ construction, etc.}

Our conventions are completely compatible with those of [10] and any details omitted here are explained there. The same is hopefully true of the references. We recall a few relevant points

- The grassmann parity is determined by the sum of all relevant degrees modulo 2. For example, when we expand the degree-zero superfield in (3.12)

$$
\boldsymbol{x}^{\mu}(\sigma, d \sigma)=x_{0}^{\mu}(\sigma)+x_{\alpha}^{\mu}(\sigma) d \sigma^{\alpha}+\ldots
$$

we have $x_{\alpha}^{\mu} d \sigma^{\alpha}=-d \sigma^{\alpha} x_{\alpha}^{\mu}$ since $d \sigma$ has degree $\operatorname{deg}\left(\sigma^{\alpha}\right)+1=1$ and $x_{\alpha}^{\mu}$ has degree $\operatorname{deg} x_{\alpha}^{\mu}=-1$. Similarly, if $\zeta$ is an odd-degree coordinate (as in the case of the M5 brane), then $d \zeta$ commutes with everything. If we had honest fermion fields $\theta$, then $d \theta$ would be bosonic, as would the 1 -form component field $\theta_{\alpha}(\sigma)$ in a superfield expansion as above.

- All vector fields are left derivations. This means for a derivation $X$

$$
X(f g)=X(f) g+(-1)^{f X} f X(g) .
$$

Since the exterior/de Rham derivative $d$ and contraction $\iota_{X}$ can both be seen as vector fields on the shifted tangent bundle $T[1] M$, we assume they also are left derivations. They are defined by

$$
d f=d z^{a} \frac{\partial f}{\partial z^{a}}, \quad \iota_{X} d f=X(f) .
$$

(In [10] we wrote $X \cdot f$ for $X(f)$. It is unclear why that seemed like a good idea.)

- The only exception to the above rule is the left argument of any Poisson bracket, which is a right derivation. Therefore, if $(\bullet, \bullet)$ is the degree $-P$ bracket on target space $\mathcal{M}$,

$$
(f, g h)=(f, g) h+(-1)^{(f-P) g} g(f, h), \quad(g h, f)=g(h, f)+(-1)^{(f-P) h}(g, f) h .
$$


Incidentally, the "right derivatives" of [10] defined by $d f=\partial_{a}^{R} f d z^{a}$ (for $f=f(z)$ ) are not right derivations in the above sense. (Fortunately, our commitment to left derivations was strong even in 2018, so, to our knowledge, none of the formulas in [10] contain any errors.)

- A graded symplectic form $\omega$ and its Poisson bracket $(\bullet, \bullet)$ are related by

$$
\iota_{X_{f}} \omega=(-1)^{f} d f, \quad X_{f} \equiv(f, \bullet) .
$$

If $\Sigma$ is a real (orientable) manifold, we identify its complex of polyforms $\Lambda^{\bullet} \Sigma$ with the ring of functions over the shifted tangent bundle $T[1] \Sigma$. We mention this here to fix our convention with regard to the wedge product: if $\alpha$ is a $p$-form

$$
\alpha=(p !)^{-1} \alpha_{\alpha_{1} \alpha_{2} \ldots \alpha_{p}} d \sigma^{\alpha_{1}} \cdots d \sigma^{\alpha_{p}}
$$

and $\beta$ is a $q$-form, the wedge product is simply

$$
\alpha \wedge \beta=\alpha \beta=(p !)^{-1}(q !)^{-1} \alpha_{\alpha_{1} \alpha_{2} \ldots \alpha_{p}} \beta_{\alpha_{p+1} \cdots \alpha_{p+q}} d \sigma^{\alpha_{1}} \cdots d \sigma^{\alpha_{p+q}},
$$

so the wedge product in terms of the antisymmetric $p$ - and $q$-tensor components acquires the standard factor of $\left(\begin{array}{c}p+q \\ p\end{array}\right)$.

We need some formulas for an AKSZ construction where the source manifold $\mathcal{N}$ has a measure of non-standard degree

$$
-n=\operatorname{deg} \int_{\mathcal{N}}
$$

as in the extension of AKSZ topological field theory to strata of arbitrary dimension of ([76], Remark 6.4). We assume that the measure "acts from the right", so there are no minus signs when pulling odd-parity constants out of integrals from the left. For the case $\mathcal{N}=T[1] \Sigma$ employed in the main test, this means that in the left-hand side of (3.10) the fermionic integral is given by iterated right Grassmann derivatives $\partial^{\text {right }} / \partial\left(d \sigma^{\alpha}\right)$.

In all cases, we have a non-degenerate Poisson structure $[\bullet, \bullet]$ on the $\operatorname{space} \operatorname{Maps}(\mathcal{N} \rightarrow$ $\mathcal{M})$ given by transgressing the target space symplectic form $\omega$ to a symplectic form $\Omega$ on the space of maps:

$$
\omega=\frac{1}{2} d z^{a} \omega_{a b}(z) d z^{b} \rightarrow \Omega=\int_{\mathcal{N}} \frac{1}{2} \delta \boldsymbol{z}^{a} \omega_{a b}(\boldsymbol{z}) \delta \boldsymbol{z}^{b},
$$

where $\boldsymbol{z}^{a}=\varphi^{\star}\left(z^{a}\right)$ are the superfields that define a point $\varphi \in \operatorname{Maps}(\mathcal{N} \rightarrow \mathcal{M})$. Since $\operatorname{deg} \Omega=P-n$, we find $\operatorname{deg}[\bullet, \bullet]=n-P$. This leads to the following two interesting cases for $n$ :

$$
n= \begin{cases}P+1: \operatorname{deg}[\bullet, \bullet]=+1 & (\text { standard AKSZ) } \\ P-1: \operatorname{deg}[\bullet, \bullet]=-1 & (\text { brane phase space construction of this paper })\end{cases}
$$


We write $\delta$ instead of $d$ for the exterior derivative on $\operatorname{Maps}(\mathcal{N} \rightarrow \mathcal{M})$. This is a left derivation. It defines the (left) variational derivative on functionals $F[\boldsymbol{z}] \in C^{\infty}(\operatorname{Maps}(\mathcal{N} \rightarrow$ $\mathcal{M})$ per our general convention:

$$
\delta F=\int_{\mathcal{N}} \delta \boldsymbol{z}^{a} \frac{\delta F}{\delta \boldsymbol{z}^{a}}
$$

The Poisson bracket of two functionals reads

$$
[F, G] \equiv X_{F} \cdot G \equiv(-1)^{F} \int(-1)^{(a+1)(F+n)} \frac{\delta F}{\delta \boldsymbol{z}^{a}} \omega^{a b}(\boldsymbol{z}) \frac{\delta G}{\delta \boldsymbol{z}^{b}},
$$

where the Poisson bivector on target space is defined by $\omega^{a b} \omega_{b c}=\delta_{c}^{a}$. The sign factor arises because $\operatorname{deg} \delta F / \delta \boldsymbol{z}^{a}=\operatorname{deg} F-\operatorname{deg} z^{a}+n$; it turns the left derivation into a right derivation, so the Poisson bracket $[\bullet, \bullet]$ is a right derivation on its left argument. Note that if we take $\mathcal{N}=\{$ point $\}, n=0$ this formula reduces to the explicit expression for the target space Poisson bracket $(\bullet, \bullet)$ in terms of its bivector.

We can now calculate the bracket of currents (1.10) that we need in the main text:

$$
\begin{aligned}
{[\langle f \mid \epsilon\rangle,\langle g \mid \eta\rangle] } & =(-1)^{f+\epsilon+n} \int(-1)^{(a+1)(f+\epsilon)} \partial_{a} f \epsilon \omega^{a b} \partial_{b} g \eta \\
& =(-1)^{f+\epsilon+n} \int(-1)^{(a+1) f+\epsilon(f+1)} \epsilon \partial_{a} f \omega^{a b} \partial_{b} g \eta \\
& =(-1)^{n+\epsilon f} \int \epsilon(f, g) \eta \\
& =(-1)^{n+(g+P) \epsilon} \int(f, g) \epsilon \eta=(-1)^{P+n+(g+P) \epsilon}\langle(f, g) \mid \epsilon \eta\rangle
\end{aligned}
$$

Lifts of vector fields. Clearly diffeomorphisms of both $\mathcal{M}$ and $\mathcal{N}$ act on the mapping space $\operatorname{Maps}(\mathcal{N} \rightarrow \mathcal{M})$, and their actions commute. Infinitesimally speaking for any vector field $X$ on $\mathcal{M}$ and $N$ on $\mathcal{N}$ we obtain their lifts $\bar{X}$ and $\bar{N}$ at the point $\varphi \in \operatorname{Maps}(\mathcal{N} \rightarrow \mathcal{M})$ by the explicit formulas

$$
\bar{N}_{\varphi}(F) \equiv \int_{\mathcal{N}} N\left(\boldsymbol{z}^{a}\right) \frac{\delta F}{\delta \boldsymbol{z}^{a}}=\int_{\mathcal{N}} N\left(\varphi^{\star} z^{a}\right)\left(\frac{\delta F}{\delta \boldsymbol{z}^{a}}\right)_{\varphi}
$$

and

$$
\bar{X}_{\varphi}(F) \equiv \int_{\mathcal{N}} X^{a}(\boldsymbol{z}) \frac{\delta F}{\delta \boldsymbol{z}^{a}}=\int_{\mathcal{N}} \varphi^{\star}\left(X^{a}(z)\right)\left(\frac{\delta F}{\delta \boldsymbol{z}^{a}}\right)_{\varphi} .
$$

The lift map is a homomorphism of graded Lie algebras for vectors $X_{1,2}$ on the target with respect to the graded commutator of vector fields

$$
[X, Y] \equiv X Y-(-1)^{X Y} Y X
$$

but an antihomomorphism for $N_{1,2}$ vectors on the source $\mathcal{N}$ ([30], Proposition 1):

$$
\overline{\left[X_{1}, X_{2}\right]}=\left[\bar{X}_{1}, \bar{X}_{2}\right], \quad \overline{\left[N_{1}, N_{2}\right]}=-\left[\bar{N}_{1}, \bar{N}_{2}\right] .
$$


The Lie commutator of source vector fields is used in the derivation of the spatial diffeomorphism generators in the main text. This minus sign can be derived by carefully calculating via (A.17).

Open Access. This article is distributed under the terms of the Creative Commons Attribution License (CC-BY 4.0), which permits any use, distribution and reproduction in any medium, provided the original author(s) and source are credited.

\section{References}

[1] W. Siegel, Two vierbein formalism for string inspired axionic gravity, Phys. Rev. D 47 (1993) 5453 [hep-th/9302036] [INSPIRE].

[2] A. Alekseev and T. Strobl, Current algebras and differential geometry, JHEP 03 (2005) 035 [hep-th/0410183] [INSPIRE].

[3] T.J. Courant, Dirac manifolds, Trans. Am. Math. Soc. 319 (1990) 631.

[4] Z.-J. Liu, A. Weinstein and P. Xu, Manin triples for lie bialgebroids, dg-ga/9508013.

[5] P. Ševera, Letters to Alan WEinstein about Courant algebroids, arXiv:1707.00265 [INSPIRE].

[6] M. Alexandrov, A. Schwarz, O. Zaboronsky and M. Kontsevich, The Geometry of the master equation and topological quantum field theory, Int. J. Mod. Phys. A 12 (1997) 1405 [hep-th/9502010] [INSPIRE].

[7] N. Ikeda and X. Xu, Current Algebras from DG Symplectic Pairs in Supergeometry, arXiv:1308.0100 [INSPIRE].

[8] N. Ikeda and K. Koizumi, Current Algebras and QP Manifolds, Int. J. Geom. Meth. Mod. Phys. 10 (2013) 1350024 [arXiv:1108.0473] [INSPIRE].

[9] M.A. Grigoriev, A.M. Semikhatov and I.Y. Tipunin, Becchi-Rouet-Stora-Tyutin formalism and zero locus reduction, J. Math. Phys. 42 (2001) 3315 [hep-th/0001081] [InSPIRE].

[10] A.S. Arvanitakis, Brane Wess-Zumino terms from AKSZ and exceptional generalised geometry as an $L_{\infty}$-algebroid, Adv. Theor. Math. Phys. 23 (2019) 1159 [arXiv:1804.07303] [INSPIRE].

[11] G. Bonelli and M. Zabzine, From current algebras for p-branes to topological M-theory, JHEP 09 (2005) 015 [hep-th/0507051] [InSPIRE].

[12] M. Hatsuda, S. Sasaki and M. Yata, Five-brane current algebras in type-II string theories, JHEP 03 (2021) 298 [arXiv: 2011.13145] [INSPIRE].

[13] M. Hatsuda and K. Kamimura, M5 algebra and SO(5,5) duality, JHEP 06 (2013) 095 [arXiv: 1305.2258] [INSPIRE].

[14] M. Hatsuda and T. Kimura, Canonical approach to Courant brackets for D-branes, JHEP 06 (2012) 034 [arXiv:1203.5499] [INSPIRE].

[15] Y. Sakatani and S. Uehara, Non-Abelian U-duality for membranes, PTEP 2020 (2020) 073B01 [arXiv: 2001.09983] [INSPIRE].

[16] A. Deser and C. Sämann, Extended Riemannian Geometry I: Local Double Field Theory, arXiv: 1611.02772 [INSPIRE]. 
[17] M.A. Heller, N. Ikeda and S. Watamura, Unified picture of non-geometric fluxes and T-duality in double field theory via graded symplectic manifolds, JHEP 02 (2017) 078 [arXiv: 1611.08346] [INSPIRE].

[18] A. Chatzistavrakidis, L. Jonke, F.S. Khoo and R.J. Szabo, Double Field Theory and Membrane Sigma-Models, JHEP 07 (2018) 015 [arXiv: 1802.07003] [INSPIRE].

[19] Y. Sakatani and S. Uehara, Exceptional M-brane sigma models and $\eta$-symbols, PTEP 2018 (2018) 033B05 [arXiv:1712.10316] [INSPIRE].

[20] Y. Sakatani and S. Uehara, Born sigma model for branes in exceptional geometry, PTEP 2020 (2020) 073B05 [arXiv: 2004.09486] [INSPIRE].

[21] W.D. Linch and W. Siegel, F-theory with Worldvolume Sectioning, JHEP 04 (2021) 022 [arXiv: 1503.00940] [INSPIRE].

[22] W.D. Linch, III and W. Siegel, F-theory from Fundamental Five-branes, JHEP 02 (2021) 047 [arXiv: 1502.00510] [INSPIRE].

[23] D. Osten, Currents, charges and algebras in exceptional generalised geometry, JHEP 06 (2021) 070 [arXiv : 2103.03267] [InSPIRE].

[24] C. Strickland-Constable, Classical worldvolumes as generalised geodesics, arXiv:2102.00555 [INSPIRE].

[25] J. Berkeley, D.S. Berman and F.J. Rudolph, Strings and Branes are Waves, JHEP 06 (2014) 006 [arXiv: 1403.7198] [INSPIRE].

[26] D.S. Berman and F.J. Rudolph, Strings, Branes and the Self-dual Solutions of Exceptional Field Theory, JHEP 05 (2015) 130 [arXiv:1412.2768] [INSPIRE].

[27] P. Ševera, Some title containing the words "homotopy" and "symplectic", e.g. this one, math/0105080.

[28] P. Ritter and C. Sämann, Automorphisms of Strong Homotopy Lie Algebras of Local Observables, arXiv:1507.00972 [INSPIRE].

[29] A. Konechny and A.S. Schwarz, On $(\kappa+l \mid q)$ dimensional supermanifolds, Lect. Notes Phys. 509 (1998) 201 [hep-th/9706003] [INSPIRE].

[30] T.T. Voronov, Vector fields on mapping spaces and a converse to the AKSZ construction, arXiv:1211.6319.

[31] C. Sämann and R.J. Szabo, Groupoids, Loop Spaces and Quantization of 2-Plectic Manifolds, Rev. Math. Phys. 25 (2013) 1330005 [arXiv:1211.0395] [INSPIRE].

[32] D. Roytenberg, On the structure of graded symplectic supermanifolds and Courant algebroids, in Workshop on Quantization, Deformations, and New Homological and Categorical Methods in Mathematical Physics, Manchester U.K. (2002) [math/0203110] [INSPIRE].

[33] N. Ikeda and K. Uchino, QP-Structures of Degree 3 and $4 D$ Topological Field Theory, Commun. Math. Phys. 303 (2011) 317 [arXiv:1004.0601] [InSPIRE].

[34] M. Grützmann, H-twisted lie algebroids, J. Geom. Phys. 61 (2011) 476 [arXiv:1005.5680].

[35] Z. Kokenyesi, A. Sinkovics and R.J. Szabo, AKSZ Constructions for Topological Membranes on $G_{2}$-Manifolds, Fortsch. Phys. 66 (2018) 1800018 [arXiv: 1802.04581] [INSPIRE].

[36] A.S. Arvanitakis and E. Malek, in preparation.

[37] D. Baraglia and P. Hekmati, Transitive Courant Algebroids, String Structures and T-duality, Adv. Theor. Math. Phys. 19 (2015) 613 [arXiv:1308.5159] [INSPIRE]. 
[38] A.S. Arvanitakis, C.D.A. Blair and D.C. Thompson, A QP perspective on topology change in Poisson-Lie T-duality, arXiv:2110.08179 [INSPIRE].

[39] M. Cederwall, G. Ferretti, B.E.W. Nilsson and A. Westerberg, Higher dimensional loop algebras, nonAbelian extensions and p-branes, Nucl. Phys. B 424 (1994) 97 [hep-th/9401027] [INSPIRE].

[40] B. Jurčo, P. Schupp and J. Vysoky, p-Brane Actions and Higher Roytenberg Brackets, JHEP 02 (2013) 042 [arXiv: 1211.0814] [INSPIRE].

[41] M. Kontsevich, Rozansky-Witten invariants via formal geometry, Compos. Math. 115 (1999) 115 [dg-ga/9704009].

[42] J. Qiu and M. Zabzine, On the AKSZ formulation of the Rozansky-Witten theory and beyond, JHEP 09 (2009) 024 [arXiv:0906.3167] [INSPIRE].

[43] O. Gwilliam and B.R. Williams, Higher Kac-Moody algebras and symmetries of holomorphic field theories, arXiv:1810.06534 [INSPIRE].

[44] T. Voronov, Higher derived brackets and homotopy algebras, J. Pure Appl. Algebra 202 (2005) 133 [math/0304038].

[45] M. Grützmann and T. Strobl, General Yang-Mills type gauge theories for p-form gauge fields: From physics-based ideas to a mathematical framework or From Bianchi identities to twisted Courant algebroids, Int. J. Geom. Meth. Mod. Phys. 12 (2014) 1550009 [arXiv: 1407.6759] [INSPIRE].

[46] M. Zabzine, Hamiltonian perspective on generalized complex structure, Commun. Math. Phys. 263 (2006) 711 [hep-th/0502137] [INSPIRE].

[47] E. Bergshoeff, D.P. Sorokin and P.K. Townsend, The M5-brane Hamiltonian, Nucl. Phys. B 533 (1998) 303 [hep-th/9805065] [inSPIRE].

[48] I. Bengtsson and A. Kleppe, On chiral p forms, Int. J. Mod. Phys. A 12 (1997) 3397 [hep-th/9609102] [INSPIRE].

[49] P. Pires Pacheco and D. Waldram, M-theory, exceptional generalised geometry and superpotentials, JHEP 09 (2008) 123 [arXiv:0804.1362] [INSPIRE].

[50] C.M. Hull, Generalised Geometry for M-theory, JHEP 07 (2007) 079 [hep-th/0701203] [INSPIRE].

[51] A. Coimbra, C. Strickland-Constable and D. Waldram, $E_{d(d)} \times \mathbb{R}^{+}$generalised geometry, connections and M-theory, JHEP 02 (2014) 054 [arXiv:1112.3989] [INSPIRE].

[52] A. Ashmore and D. Waldram, Exceptional Calabi-Yau spaces: the geometry of $\mathcal{N}=2$ backgrounds with flux, Fortsch. Phys. 65 (2017) 1600109 [arXiv:1510.00022] [InSPIRE].

[53] L. Mezincescu and P.K. Townsend, DBI in the IR, J. Phys. A 53 (2020) 044002 [arXiv: 1907.06036] [INSPIRE].

[54] M. Hatsuda and K. Kamimura, SL(5) duality from canonical M2-brane, JHEP 11 (2012) 001 [arXiv: 1208.1232] [INSPIRE].

[55] D.S. Berman and M.J. Perry, Generalized Geometry and M-theory, JHEP 06 (2011) 074 [arXiv: 1008.1763] [INSPIRE].

[56] J. Grabowski, Courant-nijenhuis tensors and generalized geometries, math/0601761.

[57] C. Córdova, T.T. Dumitrescu and K. Intriligator, Exploring 2-Group Global Symmetries, JHEP 02 (2019) 184 [arXiv:1802.04790] [INSPIRE]. 
[58] C.M. Hull and B.J. Spence, The Geometry of the gauged sigma model with Wess-Zumino term, Nucl. Phys. B 353 (1991) 379 [InSPIRE].

[59] J.A. De Azcarraga, J.M. Izquierdo and P.K. Townsend, Classical anomalies of supersymmetric extended objects, Phys. Lett. B 267 (1991) 366 [INSPIRE].

[60] D. Tennyson and D. Waldram, Exceptional complex structures and hypermultiplet moduli of $\mathbb{R}^{4,1}$ solutions of $M$-theory, JHEP 08 (2021) 088 [arXiv:2104.09900] [INSPIRE].

[61] A. Ashmore, C. Strickland-Constable, D. Tennyson and D. Waldram, Generalising $G_{2}$ geometry: involutivity, moment maps and moduli, JHEP 01 (2021) 158 [arXiv:1910.04795] [INSPIRE].

[62] C.D.A. Blair, Open exceptional strings and D-branes, JHEP 07 (2019) 083 [arXiv: 1904.06714] [INSPIRE].

[63] H. Bursztyn, N. Martinez Alba and R. Rubio, On higher dirac structures, Int. Math. Res. Not. 2019 (2019) 1503 [arXiv:1611.02292].

[64] A. Coimbra and C. Strickland-Constable, Supersymmetric Backgrounds, the Killing Superalgebra, and Generalised Special Holonomy, JHEP 11 (2016) 063 [arXiv:1606.09304] [INSPIRE].

[65] A. Coimbra, C. Strickland-Constable and D. Waldram, Supersymmetric Backgrounds and Generalised Special Holonomy, Class. Quant. Grav. 33 (2016) 125026 [arXiv:1411.5721] [INSPIRE].

[66] M. Gualtieri, Generalized complex geometry, Ph.D. thesis, Oxford U., 2003. math/0401221 [INSPIRE].

[67] A.S. Arvanitakis, O. Hohm, C. Hull and V. Lekeu, Homotopy Transfer and Effective Field Theory I: Tree-level, arXiv:2007.07942 [INSPIRE].

[68] D. Baraglia, Leibniz algebroids, twistings and exceptional generalized geometry, J. Geom. Phys. 62 (2012) 903 [arXiv:1101.0856] [INSPIRE].

[69] M. Cederwall and J. Palmkvist, $L_{\infty}$ Algebras for Extended Geometry from Borcherds Superalgebras, Commun. Math. Phys. 369 (2019) 721 [arXiv:1804.04377] [InSPIRE].

[70] Y. Cagnacci, T. Codina and D. Marques, $L_{\infty}$ algebras and Tensor Hierarchies in Exceptional Field Theory and Gauged Supergravity, JHEP 01 (2019) 117 [arXiv:1807.06028] [INSPIRE].

[71] H. Sati and U. Schreiber, Lie n-algebras of BPS charges, JHEP 03 (2017) 087 [arXiv: 1507.08692] [INSPIRE].

[72] G. Barnich, R. Fulp, T. Lada and J. Stasheff, The sh Lie structure of Poisson brackets in field theory, Commun. Math. Phys. 191 (1998) 585 [hep-th/9702176] [INSPIRE].

[73] M. Markl and S. Shnider, Differential operator endomorphisms of an Euler-Lagrange complex, Contemp. Math. 231 (1999) 177 [math/9808105].

[74] D. Fiorenza, C.L. Rogers and U. Schreiber, Higher U(1)-gerbe connections in geometric prequantization, Rev. Math. Phys. 28 (2016) 1650012 [arXiv:1304.0236] [INSPIRE].

[75] C.L. Rogers, L-infinity algebras from multisymplectic geometry, Lett. Math. Phys. 100 (2012) 29 [arXiv: 1005. 2230] [INSPIRE].

[76] A.S. Cattaneo, P. Mnev and N. Reshetikhin, Classical BV theories on manifolds with boundary, Commun. Math. Phys. 332 (2014) 535 [arXiv:1201.0290] [INSPIRE]. 